PALEO

Revue d'archéologie préhistorique

$20 \mid 2008$

Spécial table ronde ( $2^{\mathrm{e}}$ partie) : Le Gravettien : entités régionales d'une paléoculture européenne, Les Eyzies, juillet 2004

Interprétation technologique et discussion autour du statut culturel des " pièces de la Bertonne ». L'exemple de la série lithique de Seyresse (Landes, France)

Sylvain Ducasse et Mathieu Langlais

\title{
OpenEdition
}

Journals

Édition électronique

URL : http://journals.openedition.org/paleo/1674

DOI : $10.4000 /$ paleo. 1674

ISSN : 2101-0420

Éditeur

SAMRA

Édition imprimée

Date de publication : 1 décembre 2008

Pagination : 59-88

ISSN : 1145-3370

Référence électronique

Sylvain Ducasse et Mathieu Langlais, «Interprétation technologique et discussion autour du statut culturel des « pièces de la Bertonne ». L'exemple de la série lithique de Seyresse (Landes, France)», PALEO [En ligne], 20 | 2008, mis en ligne le 24 mai 2011, consulté le 07 juillet 2020. URL : http:// journals.openedition.org/paleo/1674; DOI : https://doi.org/10.4000/paleo.1674

Ce document a été généré automatiquement le 7 juillet 2020

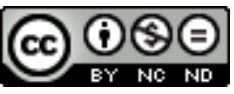

PALEO est mis à disposition selon les termes de la licence Creative Commons Attribution - Pas d'Utilisation Commerciale - Pas de Modification 4.0 International. 


\section{Interprétation technologique et discussion autour du statut culturel des « pièces de la Bertonne ». L'exemple de la série lithique de Seyresse (Landes, France)}

Sylvain Ducasse et Mathieu Langlais

\section{Introduction}

Situé en limite méridionale d'extension du Sable des Landes, le gisement de plein air de Seyresse s'inscrit dans un espace largement fréquenté par les groupes humains du Paléolithique supérieur (fig. 1), au coeur d'une zone par ailleurs marquée par l'abondance et la qualité des matériaux siliceux disponibles (Normand 1986, 2002 ; Bon et al. 1996 ; Séronie-Vivien et al. 2006 ; Chalard et al. à paraître). Découvert à la fin des années soixante par J.-P. Lescarret à l'occasion du nettoyage d'un sous-bois, le gisement fit l'objet d'une fouille de sauvetage menée par R. Arambourou et les membres du groupe d'archéologie du lycée de Dax (Arambourou 1970). Cette opération permit la mise en évidence d'une occupation paléolithique sur une superficie approchant les 80 $\mathrm{m}^{2}$. 
Figure 1 - Localisation du gisement de Seyresse (Landes) et principales occupations du Paléolithique supérieur de part et d'autre du Sable des Landes.

Figure 1 - Location of the deposit of Seyresse (Landes) and main occupations of the Upper Palaeolithic of both sides of the Sable des Landes.

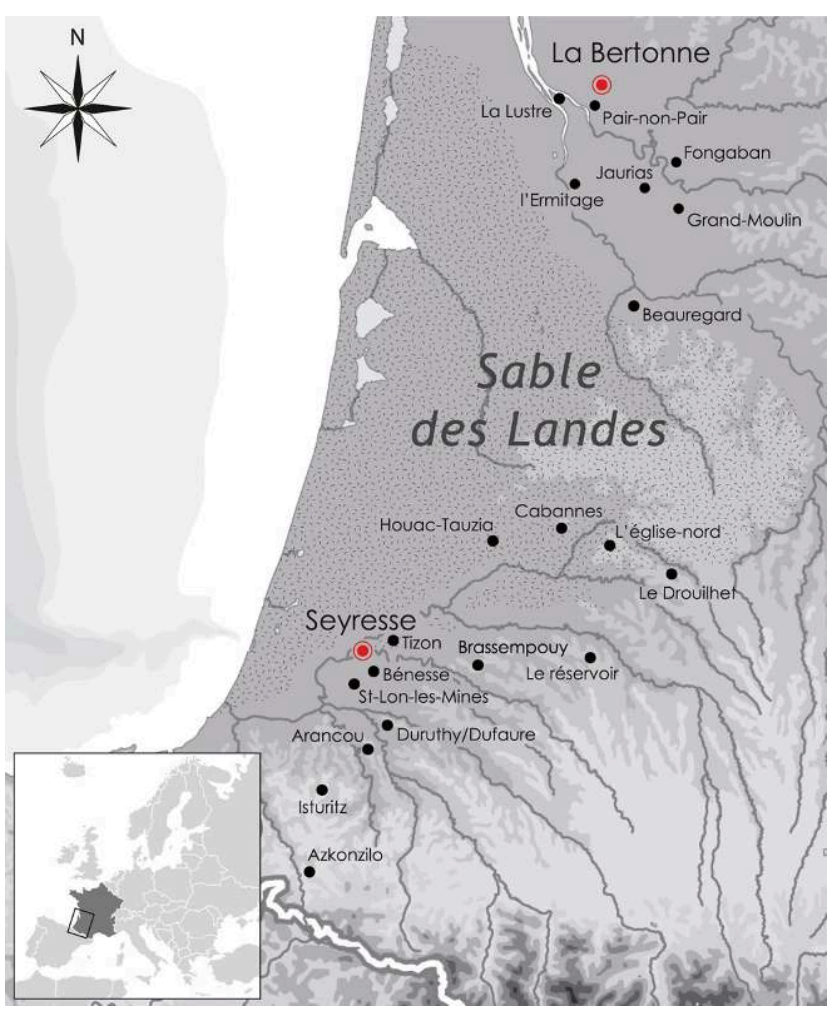

2 L'analyse typologique de l'industrie lithique ainsi recueillie, bien que peu abondante, amena R. Arambourou à attribuer le gisement de Seyresse au Proto-Solutréen (sensu Peyrony). En effet, outre la présence de types ubiquistes à l'échelle du Paléolithique supérieur, l'auteur remarque sur plusieurs pièces l'existence « (...) de retouches faites près de la base, sur la face ventrale (...) » (op. cit. p. 7), qu'il compare judicieusement aux "grattoirs de Saint-Sourd» (Leyssalles et Noone 1949) tout en les considérant comme des arguments d'attribution au Solutréen. La comparaison statistique de l'outillage de Seyresse avec l'industrie de la couche $G$ de Laugerie-Haute-Est lui permet de confirmer ce diagnostic.

3 Néanmoins, sur la base de l'analyse des éléments ayant amené R. Arambourou à privilégier la piste solutréenne, les travaux de M. Lenoir conduisirent quelques années plus tard à rediscuter de l'appartenance culturelle de l'industrie de Seyresse. Ainsi, cet auteur propose une nouvelle lecture des enlèvements plans d'extrémité isolés lors de la première publication, considérant pour sa part qu'il s'agit de " pièces de la Bertonne » équivalentes aux « pièces à retouches anormales » de F. Daleau (Daleau 1910 a et b) et aux «grattoirs de Saint-Sourd» de Leyssalles et Noone (Leyssalles et Noone 1949), véritables fossiles directeurs du Magdalénien ancien (Lenoir 1987).

4 À travers l'analyse de l'industrie de Seyresse, laquelle s'insère dans un renouvellement des recherches en technologie lithique, cet article est l'occasion de reprendre le débat concernant l'attribution des séries à pièces dites de «La Bertonne ». À la suite d'un rappel historiographique des travaux menés sur ces éléments originaux, la présentation de nos résultats aboutira, sur la base de comparaisons d'échelles diverses, à une nouvelle mise en perspective de ces industries. 


\section{1 - Un bref retour aux sources}

\section{1 - Des « compresseurs-retouchoirs » aux " pièces de La Bertonne » : petite histoire des " pièces à retouches anormales »}

5 C'est au sein d'une industrie découverte à la fin du XIX e siècle à La Bertonne (Peujard, Gironde), que F. Daleau signala pour la première fois l'existence d'un type d'objet nouveau qu'il décrit ainsi : "les retouches inverses transversales que portent ces silex sont des éclats horizontaux, longs, étroits et minces pratiqués sur le bord supérieur droit de la lame du bout opposé au conchoïde, allant tous de droite à gauche traversant tout ou partie de la face interne ; l'extrémité de ces outils souvent arrondie à la façon de la tête du grattoir classique est émoussée et non avivée par la retaille, celle-ci semble produite par la compression suivie d'un mouvement de torsion» (Daleau 1910b p. 275-276). Il nomme ces pièces «silex à retouches anormales" ou "compresseursretouchoirs ", leur conférant ainsi le statut d'outil (ibid.). Le contexte de collecte du matériel (ramassage de surface) ainsi que son apparente hétérogénéité (indices de Paléolithique moyen, supérieur et de Néolithique) n'ont par ailleurs pas permis d'attribution plus précise.

Quarante ans plus tard, et sans doute par méconnaissance des publications de F. Daleau, G. Leyssalles et H.-V.-V. Noone décrivent au Pech Saint-Sourd (Les Eyzies-de-Tayac, Dordogne) un nouvel «instrument", pourtant très proche des «silex à retouches anormales » de la station de la Bertonne : ces pièces « (...) présentent, à l'une de leurs extrémités et sur leur faceinférieure, une série de longues cannelures transversales et plus ou moins parallèles, résultantdel'enlèvement de lamelles par une pression ou percussion quelconque exercée latéralement sur laface supérieure gauche de la lame. (...) il semble donc qu'il s'agisse d'un instrument conçuspécialement pour servir de grattoir, mais dont la forme nouvelle n'a pas été signalée jusqu'àprésent »(Leyssalles et Noone 1949 - p. 251). De fait, le terme de "grattoirs de Saint-Sourd» est créé pour désigner ces éléments particuliers que les auteurs, à la différence de F. Daleau qui n'en avait pas la possibilité à son époque, attribuent à une phase moyenne de l'Aurignacien ${ }^{3}$. Ils signalent, pour finir, la présence de pièces similaires en contexte solutréen et magdalénien à Laugerie-Haute, preuve, selon eux, de la persistance du procédé.

7 Si à l'aube des années cinquante, ces « outils " particuliers - souvent comparés à des grattoirs - bénéficient d'une reconnaissance et d'une description même limitée, aucun recoupement n'est officiellement fait entre les publications citées précédemment, ceci n'arrangeant pas le flou entourant leur attribution culturelle (en grande partie lié à la nature des séries, le plus souvent issues de ramassages de surface). De fait, au moment où R. Arambourou étudie l'industrie de Seyresse et en compare certains éléments avec les " grattoirs de Saint-Sourd ", rien ne l'amène à réviser son jugement.

Alors qu'en 1973 P.-Y. Demars signale la présence de cinq "grattoirs de Saint-Sourd » à la Bombetterie (Cublac, Corrèze) au sein d'une industrie attribuée à l'Aurignacien (Demars 1973), M. Lenoir, plus d'une vingtaine d'années après les travaux de Leyssalles et Noone, reprend l'analyse du matériel récolté par F. Daleau sur le gisement de la Bertonne et établit pour la première fois un lien entre toutes ces découvertes. Offrant la première analyse détaillée de ces objets, il propose alors, non sans réserves, d'attribuer une grande part de la série au Magdalénien ancien et note, notamment, la présence de 
«nombreux éclats à retouche abrupte qui cependant ne rappellent pas les raclettes (...) » (Lenoir 1976 - p. 43). C'est donc à cette occasion que, rapprochant ces pièces des exemplaires décrits au Pech Saint-Sourd, il opte, par refus des interprétations fonctionnelles données auparavant («compresseur», "grattoir», amincissements), pour l'utilisation d'une terminologie plus neutre tout en rendant hommage au travail précurseur de Daleau : après les " compresseurs retouchoirs ", les "pièces à retouches anormales » et les "grattoirs de Saint-Sourd ", il défend l'appellation de "pièces de la Bertonne ».

Suite à une nouvelle récolte de matériel effectuée par G. Fredon sur la station éponyme, M. Lenoir publie un nouvel article dans lequel il discute plus précisément des aspects «fonctionnels » et chrono-culturels des pièces de la Bertonne. Pour cela, le corpus de gisements est largement enrichi et y figure, notamment, le site de Seyresse (Lenoir 1987). L'auteur explique l'organisation particulière des "retouches » observables sur les pièces de la Bertonne à la lueur d'expériences de taille l'ayant «(...) conduit à l'obtention presque fortuite de retouches de type la Bertonne en faisant en percussion posée sur bord d'enclume à l'aide d'un galet oblong une troncature retouchée inverse en extrémité de lame ou d'éclat épais de section triangulaire» (op. cit. p. 170). Ces éléments constitueraient donc selon lui des "pièces tronquées d'un type particulier communes dans les stades anciens du Magdalénien (...)» (ibid. p. 171).

10 Bien qu'il cite le cas des pièces "d'Orville», M. Lenoir, en même temps qu'il les distingue des pièces de La Bertonne du fait qu'elles ne comportent « (...) pour la plupart qu'un seul négatif d'enlèvement lamellaire (...)» (ibid. p.168), évacue l'idée d'une interprétation de ces pièces comme nucléus à lamelles, hypothèse alors privilégiée pour le gisement berrichon (cf. infra ; Perlès 1982). En l'absence récurrente de fraction lamellaire au sein des ensembles à " pièces de La Bertonne », il reste donc, à la suite de F. Daleau, G. Leyssalles et H.-V.-V. Noone, attaché à une interprétation typologique de ces éléments, les considérant comme des outils particuliers. "Ce sont des outils à enlèvements inverses transversaux obliques qui prolongent le plus souvent les enlèvements d'une troncature retouchée inverse. (...), ils sont presque exclusivement dextres lorsque la pièce est examinée selon sa face ventrale (...) » (Lenoir 1987 - p. 167). Au demeurant, est proposée et argumentée pour la première fois une piste d'attribution culturelle claire, celle du Magdalénien ancien, futur Badegoulien (Rigaud 1989).

\section{2 - Les pièces « d'Orville », une histoire parallèle ?}

11 Tandis que M. Lenoir pose un regard neuf sur les "pièces à retouches anormales » de Daleau (Lenoir 1976), C. Perlès réalise un sondage sur le site de plein air d'Orville (Indre) découvert quelques mois auparavant par M. Haté (Perlès 1977). Ce sondage, qui fit suite à des prospections systématiques mises en place par J. Allain au début de l'année 1975, permit de recueillir une série lithique originale, d'apparence homogène, s'ajoutant à l'abondant matériel issu du ramassage (ibid.).

12 L'originalité du matériel réside dans la présence marquée, voire dominante, d'un type d'objet n'ayant «pratiquement pas d'équivalent dans le Paléolithique français » (ibid. p. 141). "Ces pièces ont en commun non pas une morphologie mais une série d'opérations techniques: d'abord une troncature inverse, souvent déjetée sur la face d'éclatement, puis l'enlèvement d'une lamelle le long de l'arête du dièdre troncature-face d'éclatement, à partir de l'extrémité droite de la troncature lorsque la pièce est orientée sur sa face d'éclatement, 
troncature en haut »(ibid.). Si cette description ne manque pas de rappeler les « pièces de La Bertonne ", C. Perlès, tout comme M. Lenoir, effectue une distinction basée sur le degré de récurrence des enlèvements lamellaires: "les cas d'enlèvements multiples sont rares et ils n'envahissent jamais la face inférieure. C'est ce qui distingue (...) le plus nettement les pièces d'Orville des "pièces à retouches anormales" publiées par F. Daleau et réétudiées récemment par M. Lenoir » (ibid.). Réservée sur l'interprétation de ces objets, C. Perlès évoque trois possibilités dont aucune, selon elle, n'est apte à "rendre compte de l'ensemble des faits observés "(ibid. p. 144): outre l'hypothèse "outil» et sa variante consistant à y voir une "technique commune» (ibid.) d'aménagement pour des outils variés, la première des options citées est celle de nucléus à lamelles, proposition jamais clairement évoquée pour les " pièces de la Bertonne ».

13 C'est cette dernière hypothèse qui, quelques années plus tard, est finalement retenue à la suite d'une étude analytique détaillée publiée dans le cadre d'une série spéciale de la revue Studia Praehistorica Belgica (Perlès 1982). Les "pièces d'Orville" sont donc définitivement interprétées comme les déchets d'une production lamellaire originale, motivée par la recherche de supports spécifiques possédant « un pan droit facetté et un pan gauche lisse » (Perlès 1983 - p. 99) désignés plus tard sous le vocable de "lamelles d'Orville » (Parisot 1995). Cette lecture technologique est renforcée par l'existence, au sein de l'assemblage, de lamelles à dos dont les supports proviennent indubitablement des "pièces d'Orville », car présentant le pan "facetté » caractéristique ainsi que les vestiges, sur le pan opposé, de la face inférieure de la lame ou de l'éclat-support. À l'occasion de ce nouveau regard porté sur cette industrie, J. Pelegrin publie dans le même volume une approche expérimentale, décrivant en détail toutes les étapes de cette chaîne de production si particulière (débitage « systématique » ou " réplicatif » Pelegrin 1982 - p. 153).

Quant à l'attribution de la série d'Orville, si aucune piste n'était véritablement proposée au-delà d'un rattachement au Paléolithique supérieur lors de la première publication (Perlès 1977), la voie magdalénienne ouverte au début des années $80^{4}$ va finalement être privilégiée lors des études ultérieures. Ainsi, le travail universitaire de O. Parisot (Parisot 1995), dirigé par C. Perlès, J. Pelegrin et P. Bodu, va non seulement s'attacher à replacer ces nucléus au sein de l'ensemble des chaînes de productions mises en œuvre dans l'industrie, mais également permettre d'avancer de nouveaux arguments d'attribution chronoculturelle. Sur la base de la présence de quelques nucléus de type "la Marche/Rocher de la Caille » associés aux " pièces d'Orville » et, réciproquement, sur l'existence de ces dernières sur le site du Rocher de la Caille (Loire) (Deloge et Deloge 2003) $)^{5}$, O. Parisot évoque l'hypothèse d'un Magdalénien moyen comme la plus convaincante. Depuis, cette proposition a été très largement relayée, publiée à plusieurs reprises (Alix et al. 1995 ; Soriano et Lechenet 2000 ; Soriano 2004).

Si proches puissent-elles paraître à l'issue de ces descriptions, il semble en définitive que l'appréhension de l'ensemble de ces pièces à enlèvements transversaux, outre leur pluralité terminologique héritée de l'histoire et de la régionalisation des recherches, doit encore être approfondie du point de vue technologique. C'est particulièrement le cas pour les "pièces de La Bertonne » et ce, au regard de ce qui a déjà été réalisé pour les "pièces d'Orville». Ainsi seulement, pourra être discuté le bien-fondé de leur distinction. avérée fructueuse. Lors d'un séjour au dépôt du Service Régional de l'Archéologie 
d'Hasparren, en fin d'année 2005, nous avons pu recueillir des données technologiques suffisantes pour discuter du statut de ces fameuses " pièces de La Bertonne " et préciser le contexte techno-économique global de l'industrie. La proximité géographique de Seyresse avec le gisement à raclettes de Cabannes où une " pièce de la Bertonne » a pu être isolée (Gellibert et Merlet 2001; Ducasse 2004, thèse en cours ; Merlet 2005) n'est évidemment pas étrangère à ce choix, la comparaison de ces deux assemblages permettant, nous le verrons, d'alimenter le débat lié à la question de l'attribution culturelle de ces industries particulières.

\section{2 - Présentation générale de l'industrie de Seyresse}

Bien que n'ayant pas donné lieu à une étude détaillée semblable à celle que nous proposons ici pour les "pièces de La Bertonne", l'observation de l'ensemble de l'industrie a tout de même permis l'établissement d'une base de données succincte visant à obtenir une image fiable et chiffrée de sa composition. Toutefois, une attention particulière a été portée à la réalisation de remontages, ceci nous permettant de répondre à certaines questions d'ordre taphonomique et techno-économique.

\section{1 - Une industrie homogène ?}

18 Le recours aux remontages, facilité par la faible quantité de matériel (fig. $2, \mathrm{n}^{\circ} 2 ; \mathrm{N}=$ 385) a donc été privilégié pour appréhender cette série dans le but premier d'en tester l'homogénéité. Sa composition, bien que marquée par l'existence d'un tri dimensionnel lié aux méthodes de fouilles (absence de fraction fine, notamment lamellaire) est en effet, par la présence de déchets correspondant aux principales étapes de la chaîne opératoire, propice à cet exercice. Il s'agissait par ailleurs de tester, dans un second temps, les observations effectuées par R. Arambourou sur l'organisation spatiale des vestiges lithiques: "la répartition de l'outillage, comme celle des débitages et des galets montre trois concentrations : une au sud-ouest, la seconde vers le nord, la troisième à l'est [...] Les concentrations de galets paraissent correspondre aux anciens foyers. Celle des outils et des débitages les entourent généralement » (Arambourou 1970 - p. 10).

L'homogénéité de l'assemblage, dont l'apparence est renforcée par l'utilisation de matières premières peu diversifiées issues principalement des formations maestrichtiennes locales (fig. 2, $\mathrm{n}^{\circ} 3$; Normand 2002), a pu être confirmée par cette approche, non seulement sur des bases technologiques mais aussi par le biais d'une projection planimétrique des différents remontages effectués (fig. 3). Ces projections, dont la précision n'a pu être optimisée par l'utilisation des carnets de fouille restés indisponibles, ont donc été réalisées par mètre carré, superposées aux densités du matériel lithique. Seuls les galets ont pu être situés de façon absolue, R. Arambourou les ayant figurés dans sa publication (op. cit.). Si de manière générale, le plan ainsi proposé semble confirmer les observations faites par le fouilleur (concernant notamment l'existence de trois concentrations de vestiges), le traitement différentiel de chaque ensemble de matières premières renforce cette impression de structuration de l'espace. En effet, alors que la projection des silex de Tercis, de loin les plus nombreux, correspond globalement à la dispersion totale du matériel (fig. 3, en haut), la localisation d'autres matières premières laisse apparaitre l'existence d'amas distincts suggérant une bonne préservation de l'organisation spatiale des vestiges (fig. 3, au 
centre et en bas). Par ailleurs, et contrairement aux outils qui montrent une répartition régulière sur toute l'étendue du gisement, les "pièces de La Bertonne » elles, s'illustrent par une localisation préférentielle au centre de l'espace fouillé, liées à l'une des trois concentrations déjà évoquées.

Figure 2 - L'industrie lithique de Seyresse (Landes).

Figure 2 - The lithic industry of Seyresse (Landes).

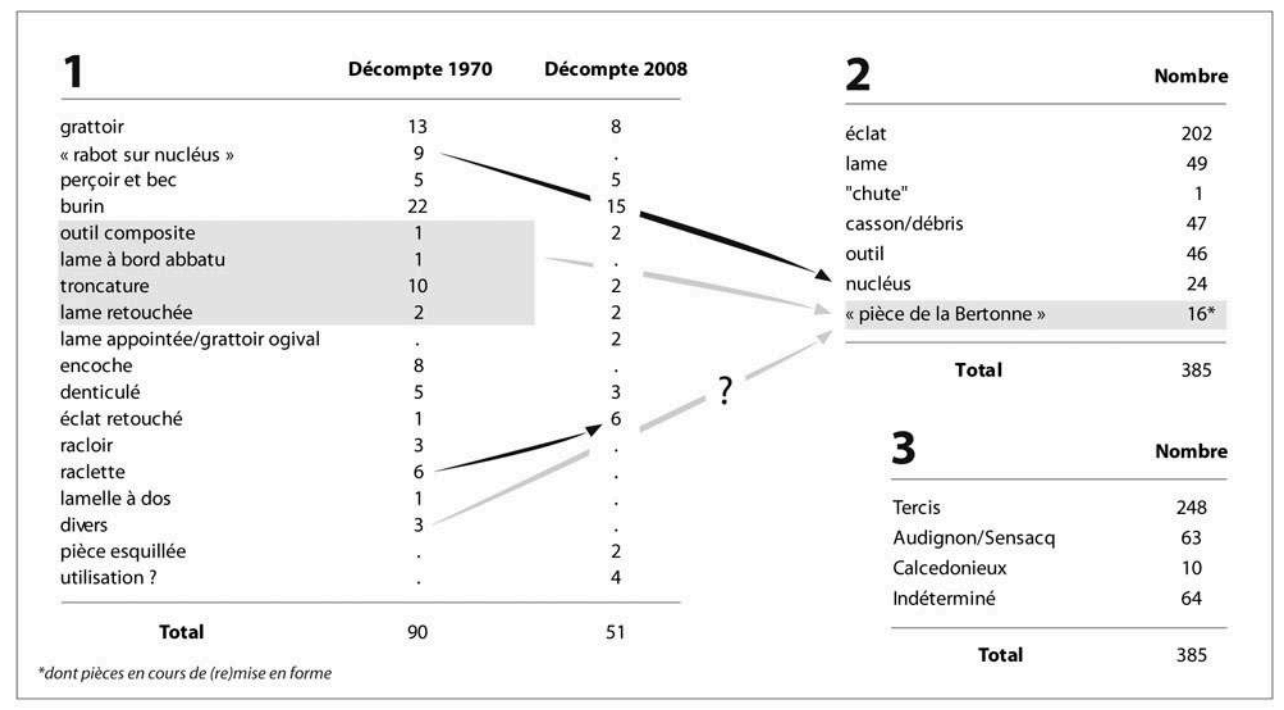

1 - décompte de l'outillage ; 2 - composition générale de l'industrie ; 3 - les différents matériaux exploités (déterminations effectuées avec l'aide de Ch. Normand).

1 - set of tools ; 2 - general composition of the industry; 3 - various exploited materials. 
Figure 3 - Répartition planimétrique du matériel lithique de Seyresse, Landes (densité par mètre carré).

Figure 3 - Spatial distribution of the lithic material of Seyresse, Landes (density per square metre).
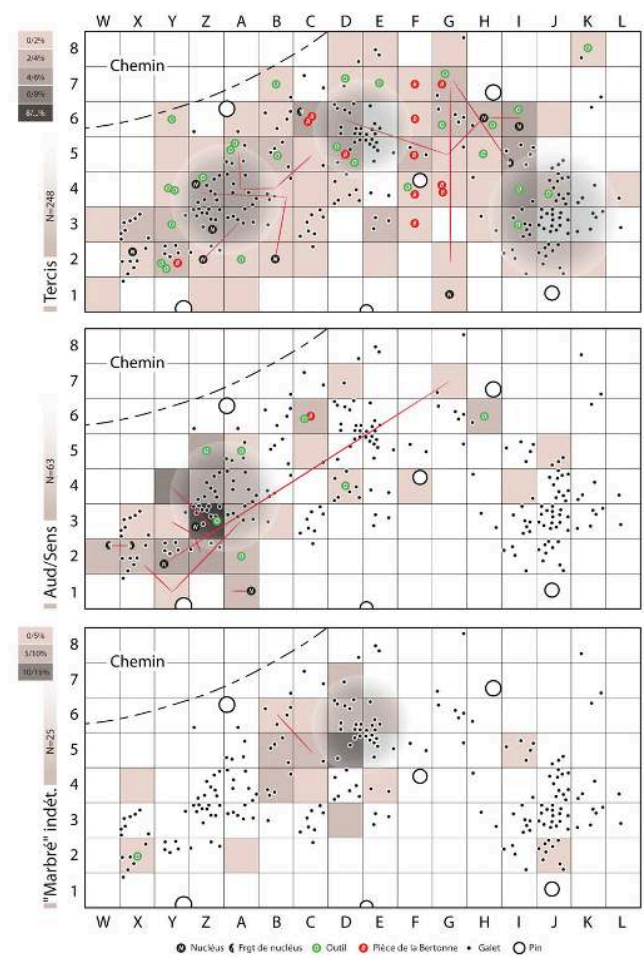

Les tracés rouges représentent les différents remontages (d'après Arambourou 1970, modifié). The red lines represent the various refits (According to Arambourou 1970, modified).

Cette approche préliminaire montre tout l'intérêt de l'analyse de ce matériel qui, bien que quantitativement réduit, offre d'intéressantes perspectives de recherches. Nous ayant permis d'en évaluer le degré d'homogénéité, les remontages effectués nous ont ensuite amenés à préciser l'identification typo-technologique de l'assemblage.

\section{2 - Aperçu typo-technologique global}

Ainsi composée de matériaux collectés dans un rayon n'excédant pas $35 \mathrm{~km}$, aucun élément allochtone n'évoquant de liens extra-régionaux (silex d'Audignon et de Sensacq pour les plus lointains, $\mathrm{N}=63$ ), cette industrie se caractérise de prime abord par l'emploi d'une gamme de supports assez diversifiée. Cette diversité, allant de l'éclat à la lame en passant par l'éclat allongé, s'exprime au sein d'un outillage dominé par les burins (fig. $4, \mathrm{n}^{\circ} 4$ bis; fig. $5, \mathrm{n}^{\circ} 2$ ) associés à des grattoirs (fig. $9, \mathrm{n}^{\circ} 2$ ), des pièces perforantes (fig. $9, n^{\circ} 4$ ), de rares outils composites (fig. $5, n^{\circ} 1$ ) ainsi qu'à quelques pièces à retouches latérales ou denticulées. Outre cet outillage, nous verrons par ailleurs qu'une partie des supports débités est réinjectée dans la sphère de production, jouant le rôle de nucléus. 
Figure 4 - Les productions lithiques de Seyresse (Landes). Figure 4 - The lithic productions of Seyresse (Landes).

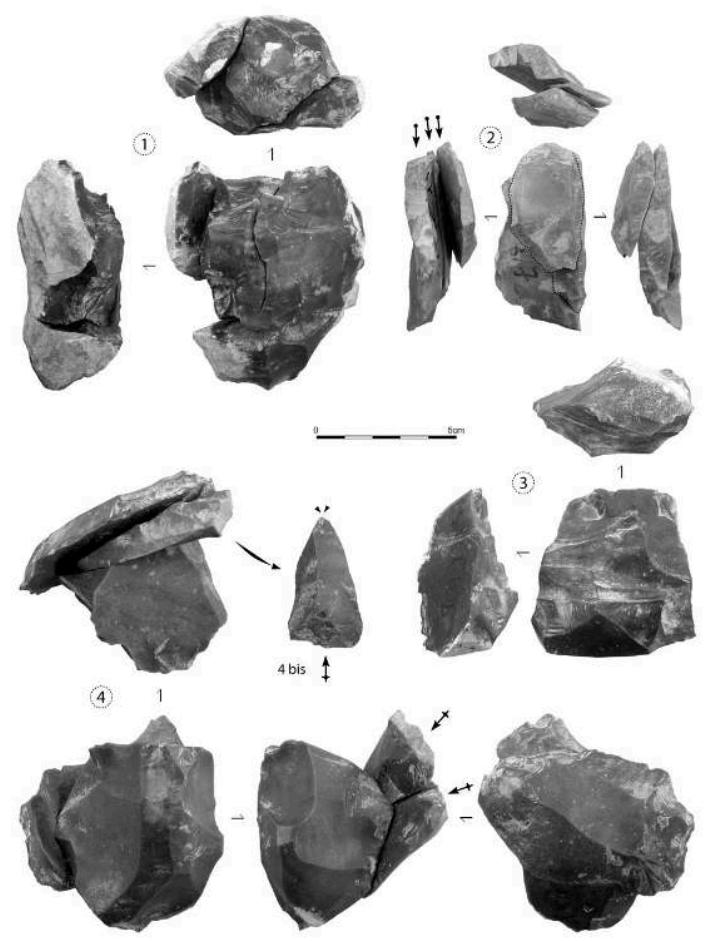

1 - nucléus à éclats ; 2 - remontage d'une séquence latérale de production laminaire ; 3 - nucléus à éclats allongés ; 4 - nucléus à architecture laminaire débité à la pierre, faisant état d'une réorientation de la production.

1 - flake core; 2 - refitting of a lateral production sequence of blades; 3 -long flakes core ; 4 - blade core knapped with mineral hammer, stating a reorientation of the production.

(clichés S. Ducasse)

(Photos S. Ducasse).

Figure 5 - Exemples d'outils (Seyresse, Landes).

Figure 5 - Examples of tools (Seyresse, Landes).

1 - grattoir/burin ; 2 - burin dièdre d'angle (percussion organique probable).

7 - end-scraper + burin (composite tool) ; 2 - lateral dihedral burin (Likely organic percussion). 

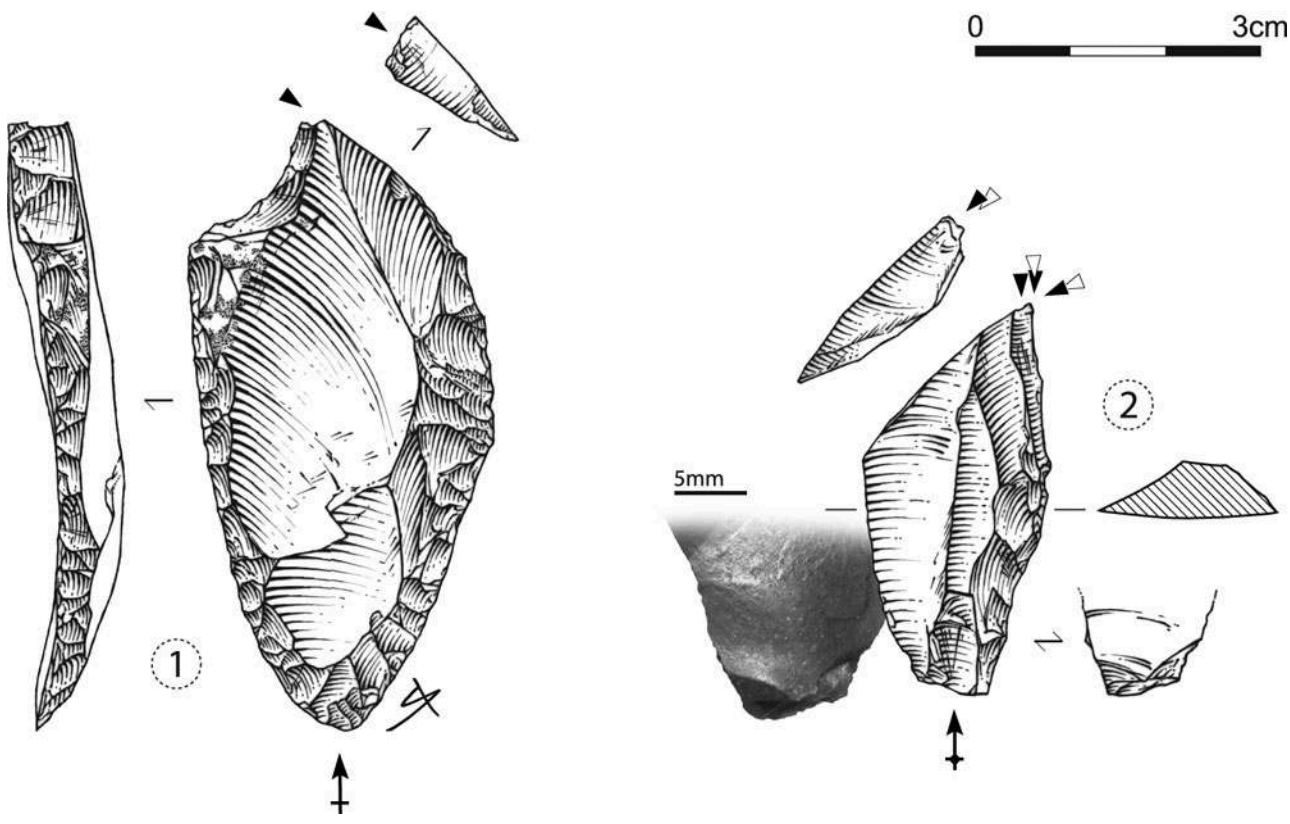

Dessins S. Ducasse.

Drawings S. Ducasse. 
Figure 6 - Les productions lithiques de Seyresse (Landes). Figure 6 - The lithic productions of Seyresse (Landes).
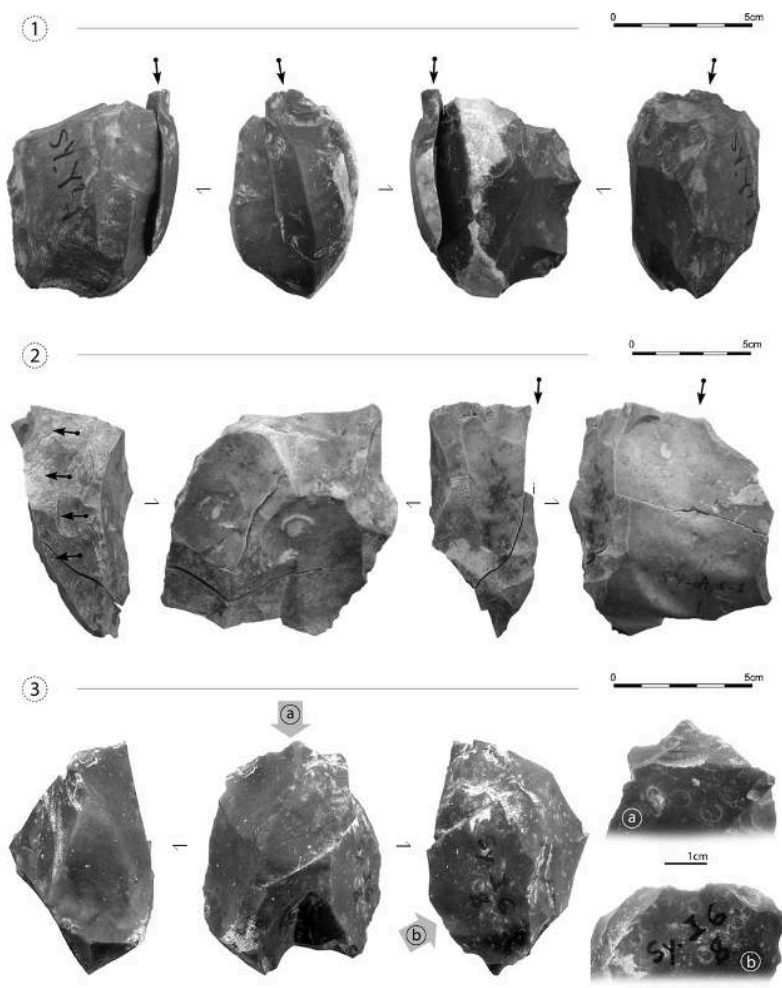

1 - nucléus à architecture laminaire (crête postérieure) débité à la pierre ; 2 - nucléus à éclats allongés sur grande tablette laminaire ; 3 - nucléus à éclats montrant de nombreux cônes incipients 1 - core in laminar shaping (posterior crest) knapped with mineral hammer; 2 - long flakes core on big core tablet; 3 - flakes core with numerous incipients cones.

(clichés S. Ducasse)

(Photos S. Ducasse). 
Figure 7 - Exemples de « pièces de la Bertonne » de Seyresse (Landes) ; débitage «plan, préférentiel, sur tranche transversale "

Figure 7 - Examples of " pièces de la Bertonne » of Seyresse (Landes) ; " plan, préférentiel, sur tranche transversale » debitage.

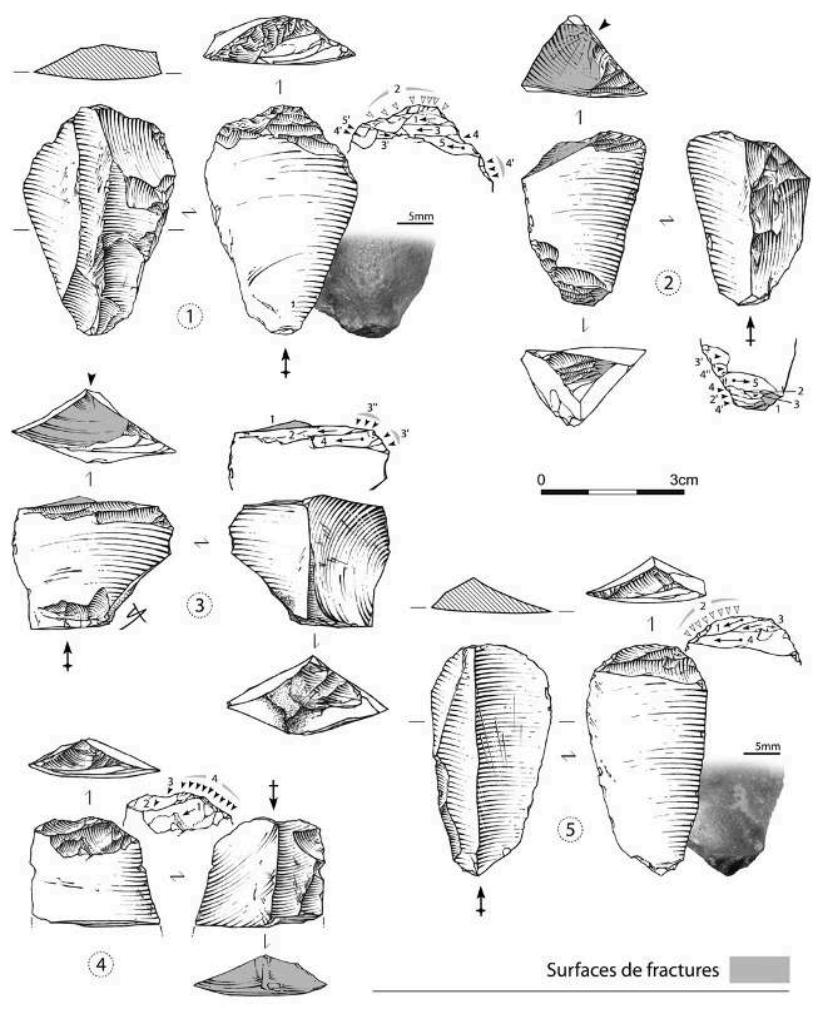

(dessins S. Ducasse).

(drawings S. Ducasse) 
Figure 8 - Exemples de « pièces de la Bertonne » de Seyresse (Landes) ; débitage «plan, préférentiel, sur tranche transversale ».

Figure 8 - Examples of " pièces de la Bertonne » of Seyresse (Landes) ; " plan, préférentiel, sur tranche transversale » debitage.

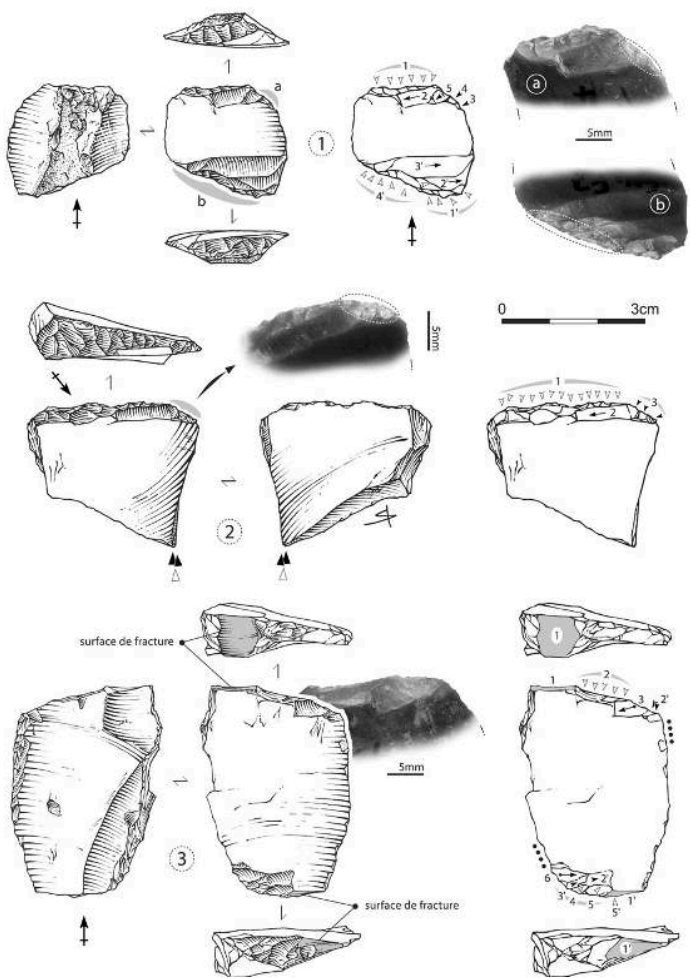

Notons la reprise proximale inverse des derniers enlèvements lamellaires ( $n^{\circ} 1$ et 2 ). Note the inverse and proximal « retouch » of the last bladelet removals ( $n^{\circ} 1$ and 2 ).

Dessins et clichés S. Ducasse.

Drawings and photos S. Ducasse. 
Figure 9 - Probables pièces mixtes « Bertonne » / outil (Seyresse, Landes) Figure 9 - Likely mixed pieces «Bertonne »/ tool (Seyresse, Landes).

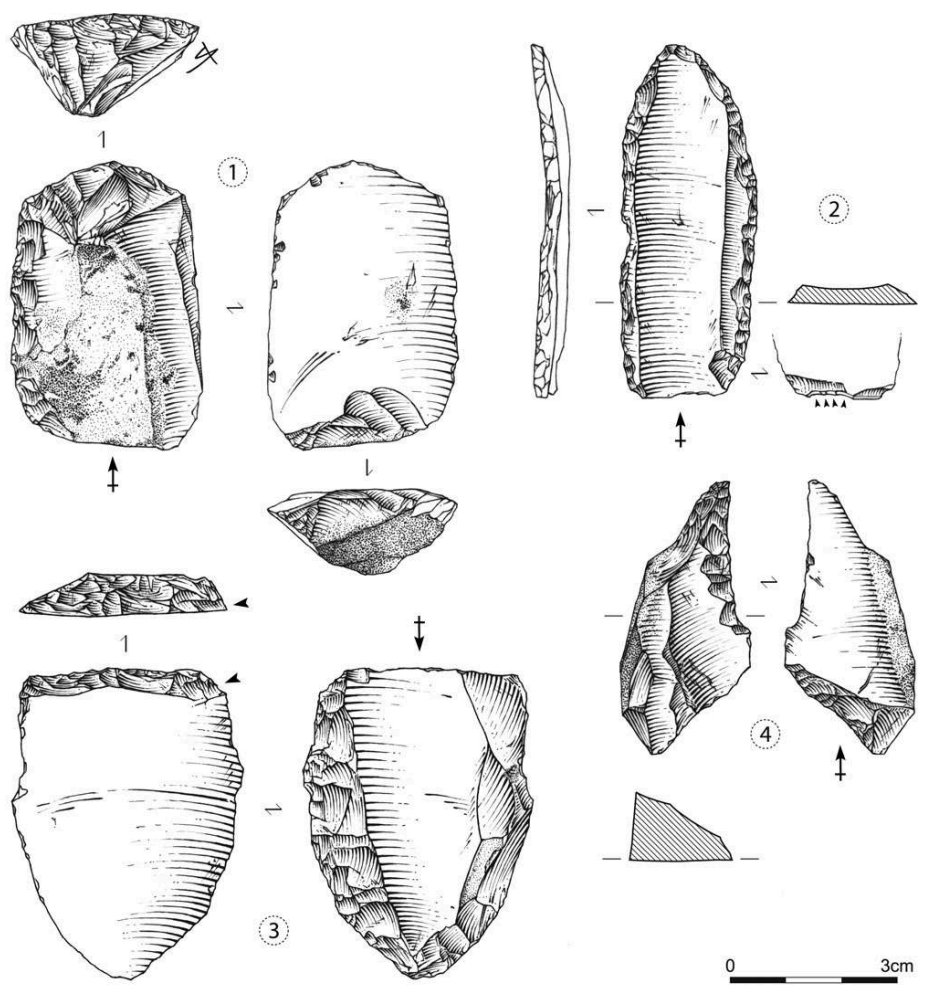

1 à 3 - grattoirs sur lames retouchées (le grattoir $n^{\circ} 1$ ne nous semble pas pouvoir être interprété comme un nucléus à lamelles) ; 4 - perçoir opposé à une troncature inverse oblique (mise en forme d'une table lamellaire?).

1 - 3 - end-scrapers on retouched blades (the end-scraper $n^{\circ} 1$ doesn't seem to be able to be interpreted as a bladelets core) ; 4 - perçoir opposite to an oblique and inverse truncation.

Dessins S. Ducasse.

Drawings S. Ducasse.

Le nombre d'outils décomptés lors de notre analyse montre certaines différences vis-àvis des données publiées, faisant état de 51 pièces contre 90 pour R. Arambourou (fig. 2, $\mathrm{n}^{\circ} 1$ ). La cause de ce décalage est double: s'il s'est avéré que certains des outils déterminés en 1970 ne présentaient aucune modification intentionnelle ( faux » coups de burin notamment), la relecture technologique de cet assemblage a, en outre, permis d'écarter certaines pièces de l'outillage, celles-ci ne correspondant finalement qu'à des déchets de débitage. Ainsi, mis à part le cas des « rabots sur nucléus » (qui de leur côté ne posent aucun problème), la réinterprétation de certains éléments en tant que "pièces de La Bertonne » (fig. 2 en grisé) devait, au vu de l'ambiguïté de leur statut, engendrer leur éviction de l'outillage en l'attente de leur analyse détaillée (cf. infra). Il existe, au demeurant, de véritables différences d'interprétation typologique entre les deux décomptes: pour ne prendre qu'un exemple, les raclettes signalées par $\mathrm{R}$. Arambourou ne nous semblent pas pouvoir être dénommées ainsi, le bord de ces outils ne présentant pas la retouche si caractéristique de ce type de pièces. Cela se laisse d'ailleurs comprendre à la lecture de l'article où l'auteur décompte « 6 raclettes sur éclats minces où une retouche très fine se développe complètement sur un bord ou, plus souvent, demeure partielle, comme sur les raclettes moustériennes » (ibid. - p. 6).

L'existence d'une variété de supports pose d'emblée la question de la pluralité des schémas opératoires. En d'autres termes, la présence concomitante de lames, dont 
certaines montrent les stigmates d'une percussion tendre organique (en relation avec des talons fréquemment facettés, fig. $7, \mathrm{n}^{\circ} 1$ et 5 ; fig. $5, \mathrm{n}^{\circ} 2$ ) d'éclats, voire d'éclats allongés qui, proches des lames par certaines caractéristiques (modules, matières premières) s'en éloignent par d'autres (régularité, préparation moins soignée, percussion à la pierre) est-elle le reflet de la mise en place de schémas opératoires distincts?

L'analyse préliminaire des nucléus, couplée à la réalisation de remontages, illustre finalement le contraire, la succession de ces différents objectifs sur un même volume paraissant plus conforme à la réalité technologique de l'assemblage. Ainsi, rien n'interdit de penser que l'intention laminaire, bien perceptible à travers l'existence de supports relativement soignés, ait pu intervenir en amont de l'exploitation de certains volumes. La présence de nucléus associant une gestion volumétrique relativement soignée (entretiens des flancs à partir de crêtes postérieures...) à l'utilisation d'une percussion à la pierre (fig. $4, \mathrm{n}^{\circ} 4$; fig. $6, \mathrm{n}^{\circ} 1$ ), semble ainsi pouvoir étayer cette hypothèse. La recherche d'éclats allongés marque nettement l'industrie (nombreux nucléus et produits; fig. $4, \mathrm{n}^{\circ} 3$; fig. $6, \mathrm{n}^{\circ} 2$ ) sans qu'il soit possible, à ce stade de l'analyse, de comprendre s'il s'agit d'une simple chute de soin ou si elle correspond à un objectif économique bien différencié de celui des lames.

Quant à certains éclats minces vraisemblablement utilisés comme tranchants naturels, ils pourraient provenir de courtes séries effectuées en fin de chaîne opératoire, l'un des nucléus à éclats témoignant clairement de l'antériorité d'une production allongée. Ce choix se distingue, d'un point de vue économique, de la recherche d'éclats minces documentée au Badegoulien, cette dernière motivant généralement la mise en place d'un schéma opératoire autonome (Morala 1993 ; Bracco et al. 2003).

C'est dans ce contexte de production aux objectifs variés, qu'un schéma opératoire ramifié a été mis en œuvre dans le but manifeste d'obtenir des supports lamellaires. Ce schéma opératoire est à l'origine des "pièces de La Bertonne " que l'analyse détaillée permet désormais d'insérer dans une perspective technologique. Ce prisme analytique nous amène donc à valider une interprétation qui, bien que maintes fois évoquée, n'a pour autant jamais été démontrée.

\section{3 - Les « pièces de la Bertonne » en question : analyse descriptive et interprétation technologique}

$\mathrm{Si}$, lors de sa publication, R. Arambourou souligne la présence marquée de pièces à retouches inverses d'extrémité, il ne crée pas pour autant de catégorie typologique spécifique. L'existence de nettes variations morphologiques au sein de ces pièces le pousse au contraire à les assimiler à des types variés (fig. 2). Il distingue par exemple : "un [...] fragment de lame large, façonné en grattoir ogival [...] [portant] une vigoureuse troncature droite [...] un fragment proximal de lame à bord abattu [...] [dont] la base a été modifiée par une vigoureuse troncature [...]. Un fragment proximal a une troncature convexe et des enlèvements à plat, transversaux qui ont supprimé bulbe et plan de frappe [...]». Il remarque que ce "[...] même genre d'enlèvements transversaux se retrouve à l'extrémité distale d'un éclat allongé et d'une lame [...], pièces très semblables à celles de Saint-Sourd »(Arambourou 1970 - p. 4-6). Les lames retouchées ne sont pas oubliées, puisqu'un « 
fragment proximal de lame large " présente des «tentatives d'enlèvements minces et à plat à partir des bords» (ibid.).

À l'image de l'approche menée par C. Perlès visant à mettre en exergue les « seuls traits les plus constants" des "Orvilles" (Perlès 1982 - p. 130), M. Lenoir put, fort de l'analyse des industries du gisement éponyme, diagnostiquer l'existence de " pièces de La Bertonne » à Seyresse (Lenoir 1987). C'est donc en dépassant les aspects strictement morphologiques, qu'il devient possible de réintégrer une partie des outils publiés par Arambourou au sein d'un ensemble d'apparence hétérogène mais de technologie similaire (fig. $2, \mathrm{n}^{\circ} 1$ et 2 ). Ainsi, les décomptes présentés ici, s'ils restent préliminaires et mériteront d'être détaillés ultérieurement (Ducasse thèse en cours), offrent une nouvelle vision de l'outillage en silex. Seize pièces ont donc été isolées sous le vocable temporaire de "pièces de La Bertonne", constituant alors un groupe où sont rassemblés des éléments se situant à divers stades de leur exploitation (12 pièces abandonnées en fin d'exploitation, trois pièces probablement abandonnées en cours de (re)mise en forme et un exemplaire incertain).

\section{1 - Le prisme technologique : pour un vocabulaire descriptif}

Douze de ces pièces ont donc fait l'objet d'une observation rigoureuse afin de décrire l'enchaînement des gestes techniques visibles sur le support, leur incidence sur l'architecture de la pièce, ceci permettant, in fine, d'en déduire le rôle. La synthèse de ces observations nous a amené à l'établissement d'un schéma opératoire qui, bien que présentant des variables d'ordre technique, semble bien répondre à une intention unique : produire des supports lamellaires (fig. 10).

La sélection préférentielle de matrices allongées (fig. 7), de section indifféremment triangulaire ou trapézoïdale, trouve sa logique dans le choix de l'orientation de la table. En effet, installée exclusivement aux extrémités du support, elle inscrit potentiellement le nombre de séquences dans la longueur de ce dernier. Localisé indifféremment en zone proximale ou distale, le nombre de tables analysables s'élève à 16 sur 12 exemplaires décomptés, trois d'entre eux comportant deux tables opposées (fig. 7, $\mathrm{n}^{\circ} 2$; fig. $8, n^{\circ} 1$ et 3 ). Bien que difficile à appréhender, la segmentation volontaire de ces volumes allongés et globalement réguliers devait permettre l'obtention de plusieurs matrices aux caractéristiques stables.

31 Cette segmentation (par percussion ou par flexion) joue, par ailleurs, un rôle important lors des phases d'ouverture ou de ré-ouverture de la table de débitage (fig. 10, 2A); la moitié d'entre elles laissant visible une ou plusieurs surfaces de fracture (fig. $7, \mathrm{n}^{\circ} 2$ et 3 ; fig. 8, n 3 ). Cependant, sur les huit cas décomptés, quatre sont repris par une «troncature " inverse d'ampleur variable (fig. 10, 2D) ce qui, de fait, ne nous permet pas de considérer que lorsque cette "troncature» est totale, elle constitue le seul aménagement effectué sur la pièce (fig. $8, \mathrm{n}^{\circ} 1$ et 2 ; fig. 10, 2B). Si, sur certains volumes, un aménagement de ce type peut s'effectuer sans préparation préalable, il est probable que dans d'autres cas, la réalisation d'une fracturation corresponde à la première étape de réalisation de cette "troncature" (fig. 8, $\mathrm{n}^{\circ}$ ). Ainsi, sans minorer le statut des fractures qui ont parfois permis à elles seules la mise en forme de la table (fig. $7, \mathrm{n}^{\circ} 3$ ), la succession de ces deux opérations pourrait être plus fréquente qu'il n'y paraît, la «troncature " venant, de façon plus ou moins importante, corriger la régularité de l'arête alors créée. Car, au-delà de choix variables, parmi lesquels le recours à un 
enlèvement «burinant » reste exceptionnel (deux cas, fig. 10, $2 \mathrm{C})^{6}$, c'est une même intention qui transparaît: la création d'une nervure exploitable à la jonction entre cette nouvelle surface et la face d'éclatement du support.

Figure 10 - Schéma idéal et synthétique du débitage " plan préférentiel sur tranche transversale » établi d'après l'analyse des « pièces de La Bertonne » de Seyresse (Landes).

Figure 10 - Ideal and synthetic scheme of the " plan préférentiel sur tranche transversale » debitage based on the analysis of the "pièces de la Bertonne » of Seyresse (Landes).

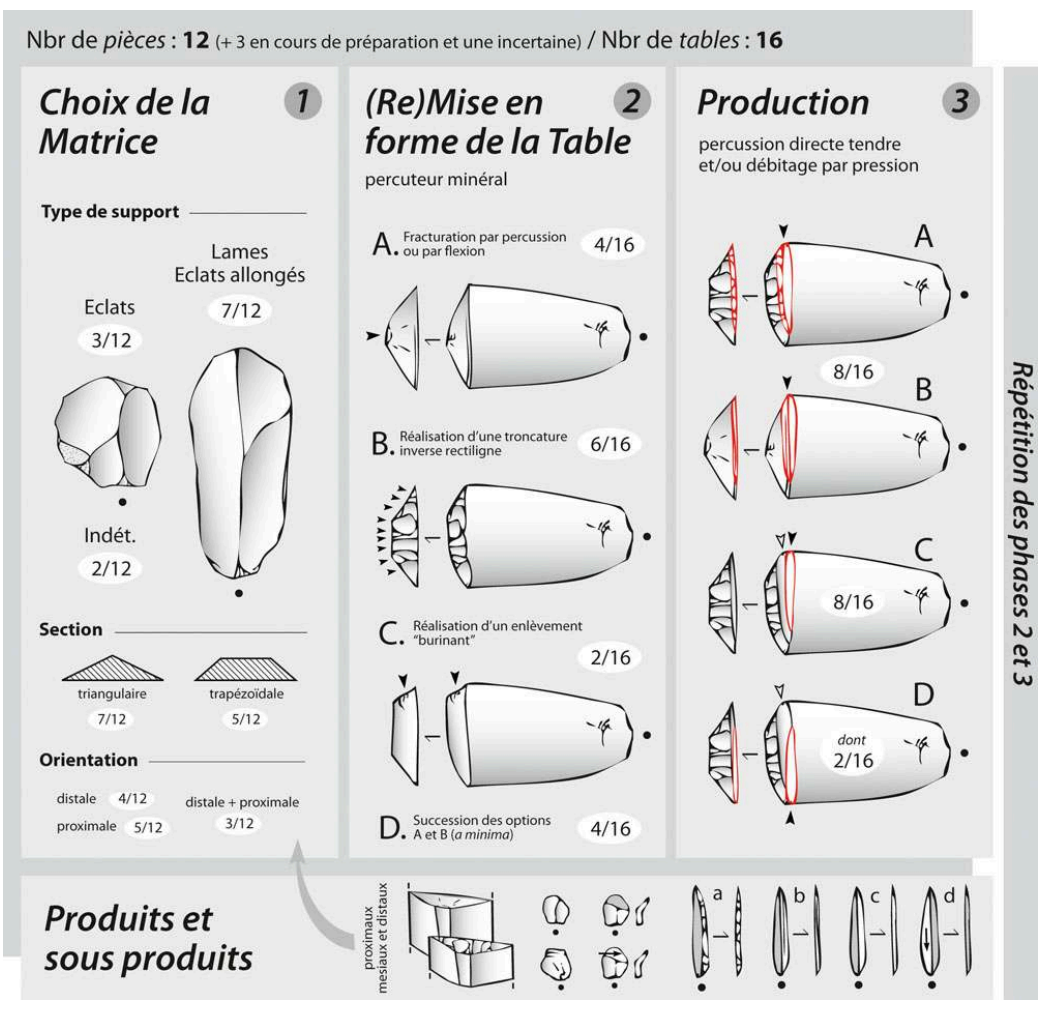

Une fois la matrice "conformée " par l'aménagement de cette nervure, le plan de frappe, lorsqu'il n'est pas simplement constitué par un pan de la face supérieure (neuf cas sur 16 ; fig. $7, \mathrm{n}^{\circ} 1$ [bord gauche] à 3 et 5 ; fig. $8, \mathrm{n}^{\circ} 1$ ) est aménagé par une reprise directe et localisée du bord latéral (fig. $7, \mathrm{n}^{\circ} 1$ [bord droit] ; fig. 8, $\mathrm{n}^{\circ}$ 3). Dans la majorité des cas uniques (deux occurrences d'enlèvements opposés), il est latéralisé à gauche, face supérieure du support tournée vers l'observateur. Lorsque les deux extrémités de la matrice ont permis l'installation d'une table, les plans de frappe sont opposés selon une diagonale proximal droit/distal gauche (fig. $7, \mathrm{n}^{\circ} 2$; fig. $8, \mathrm{n}^{\circ} 1$ et 3 ).

Ces différents aménagements menés à bien, le débitage à proprement parler peut s'engager (fig. $10-n^{\circ} 3$ ). La séquence de production consiste en l'enlèvement d'une première lamelle le long de la nervure (huit cas sur $16 ; 3 \mathrm{~A}$ et $\mathrm{B}$ ), le négatif ainsi créé pouvant donner lieu à une seconde extraction lamellaire (six cas sur $16 ; 3 \mathrm{C}$ ) voire plus rarement à une troisième (deux cas sur 16;3D). La multiplicité des enlèvements, souvent notée comme l'un des caractères permettant de distinguer les "Bertonnes " des « Orvilles» (cf. supra) n'est ici pas si évidente. La lecture diacritique montre ainsi, une fois appréhendé le rôle de chaque enlèvement, que les tentatives d'extraction lamellaire sont rarement supérieures à deux. Au demeurant, la poursuite du débitage, dont les conditions passent par un réaménagement de la table plus ou moins important, peut engendrer l'amalgame de deux séquences distinctes chronologiquement, donnant 
alors l'impression d'une plus grande récurrence (fig. 7, $\mathrm{n}^{\circ} 1$ ). En effet, les différentes séquences sont rythmées par des remises en forme systématiques, soit par fracturation volontaire de la table, soit par la réalisation directe d'une nouvelle "troncature " inverse, de sorte que les deux grandes étapes de ce schéma (mise en forme de la table / production) se répètent sur le même volume jusqu'à ce qu'intervienne une impossibilité technique. L'ensemble de ces (ré)aménagements engendre un certain nombre de déchets caractéristiques (fig. 11, en bas) dont la plupart sont absents de l'industrie de Seyresse par défaut de tamisage : divers éclats de (re)mise en forme de la troncature (présentant parfois un pan revers distal ou une portion du négatif lamellaire antérieur), tronçons de supports obtenus par percussion ou par flexion (présentant, dans le cas d'un réaménagement, les vestiges d'une ancienne table), « chutes de burin », etc.

Figure 11 - «Pièces de la Bertonne » provenant des sites de Laugerie Haute Est c. $10\left(n^{\circ} 1\right)$ et de la Malignère $\left(n^{\circ} 2\right)$, illustrant la reprise proximale inverse observée sur certaines pièces de Seyresse (d'après Demars et Laurent 1989, modifié).

Figure 11 - "Pièces de la Bertonne » from Laugerie Haute Est c.10 ( $\left.n^{\circ} 1\right)$ and La Malignère $\left(n^{\circ} 2\right)$ illustrating the inverse and proximal "retouch » observed on some pieces of Seyresse (According to Demars and Laurent 1989, modified).
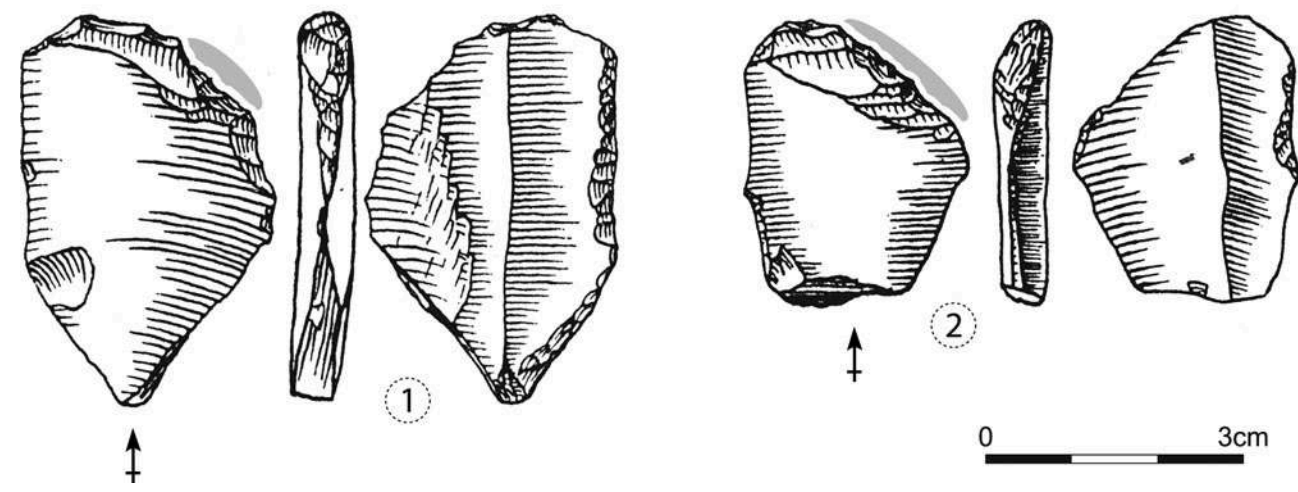

La question du mode d'extraction des lamelles recherchées reste par ailleurs ouverte: si le recours à une percussion tendre lato sensu semble concerner une partie du corpus étudié, l'hypothèse de l'emploi de la pression, parfois évoquée pour ces mêmes pièces comme un procédé de retouche (Leysalles et Noone 1949; Arambourou 1970; Lenoir 1976), a retenu notre attention au vu du caractère très "rasant" ainsi que de la régularité de certains négatifs. Loin d'être inédite au Paléolithique supérieur (Alix et al. 1995 ; Bordes et Pelegrin com. orale ${ }^{7}$ ) et déjà considérée dans le cas des "pièces d'Orville» (Pelegrin 1982; Parisot 1995), l'utilisation de la pression pour le débitage pourrait documenter une gestion nettement différenciée de chaque séquence articulant ce schéma opératoire ${ }^{8}$.

L'abandon des nucléus est majoritairement dû à des réfléchissements, conséquence fréquente d'une gestion des convexités longitudinales devenue délicate. Cependant, six fois sur seize, le dernier geste effectué sur la table de débitage consiste en une reprise inverse proximale du (ou des) dernier(s) enlèvement(s); reprise ne donnant lieu à aucune nouvelle extraction (fig. $8, \mathrm{n}^{\circ} 1$ et 2). Certaines « pièces de La Bertonne » du site éponyme (Lenoir 1983), de Laugerie-Haute-Est (Demars et Laurent 1989; Cretin 2000) ou du site de la Malignère (Demars 1985) montrent, d'après les dessins publiés, les mêmes traces de reprises proximales des tables (fig. 11). Ces stigmates pourraient correspondre, à l'image d'un ultime ravivage du plan de frappe de certains "Orvilles » 
supprimant le contre-bulbe des négatifs lamellaires (Perlès 1977 - p. 141), à une tentative avortée de remise en forme de la table par «troncature " inverse. Cette piste nous paraît être la plus raisonnable en l'attente de l'analyse d'un corpus de pièces plus étendu. Prenant alors l'allure de pièces tronquées, ces exemplaires abandonnés en cours de remise en forme ont pu être confondus avec de simples outils, légitimant une interprétation déjà appuyée par l'existence de pièces associant aux "Bertonnes " différents parties actives (Lenoir 1976 - p. 44). Ces pièces mixtes, signalées tant au Pech Saint-Sourd qu'à Orville, sont présentes à Seyresse. Ainsi, sur 12 nucléus, trois sont associés à des parties actives a priori indépendantes, correspondant exclusivement à des fronts de grattoirs (fig. $9, \mathrm{n}^{\circ} 1$ à 3 ). Il semble par ailleurs possible, sur deux d'entre eux, d'affirmer l'antériorité de l'outil sur la zone aménagée en nucléus. Une quatrième pièce associe une extrémité perforante à une troncature inverse opposée dont le rôle reste discuté (fig. 9, $\mathrm{n}^{\circ} 4$ ). Quoi qu'il en soit, ces associations ne nous semblent pas pouvoir contredire l'interprétation que nous faisons de ces pièces, considérant qu'elles illustrent un comportement économique classique visant à rentabiliser l'usage de certains supports (leur conférant une plus grande durée de vie) ${ }^{9}$.

En définitive, ce schéma opératoire, quasiment exclusif, vise à produire de fines lamelles rectilignes, parfois légèrement torses en partie proximale. La mesure de longueur maximale des tables, inscrites dans la largeur des matrices, permet de situer les modules recherchés entre 15 et $35 \mathrm{~mm}$ de long. Ces supports, absents de la série pour des raisons identiques à celles que nous évoquions concernant certains sousproduits (défaut de tamisage, tri à la fouille ?), devaient compter en leur sein des pièces à la morphologie particulière, visibles sur au moins cinq des nucléus présents dans l'assemblage. Il s'agit de lamelles associant un pan revers sénestre à un pan dextre constitué par les négatifs distaux de la troncature d'aménagement de la table (fig. 10, en bas : a). Ces lamelles à pan « cannelé » correspondent en tous points aux « lamelles d'Orville » décrites par C. Perlès (cf. supra) ${ }^{10}$.

Cette analyse, en nous permettant d'établir une description technologique neutre de l'ensemble de ces pièces, nous conduit in fine à l'élaboration d'une terminologie exempte de toute référence éponyme, nécessaire à des comparaisons objectives (Fourloubey et al. 2006; Ducasse et Langlais 2007) : les "traits les plus constants " (Perlès 1982 - p. 130) de ce schéma opératoire l'inscrivent dans la famille des débitages plans préférentiels sur tranche transversale dont l'une des principales caractéristiques réside dans le débordement des produits recherchés en face inférieure de la matrice (enlèvements plans). L'usage du terme "préférentiel », s'il peut se discuter au cas par cas, nous semble bien rendre compte de la tendance observée ainsi que de la «structure » de ce débitage.

\section{2 - Un schéma opératoire culturellement discriminant ?}

Le dynamisme des recherches menées ces dernières années sur la question des productions lamellaires, perçues comme un important vecteur identitaire car situées fréquemment au cœur des stratégies de subsistance (confection des armements), permet de renforcer le caractère original de ce procédé de débitage. En effet, les données disponibles sur les principaux technocomplexes du Paléolithique supérieur ancien montrent que ce débitage "plan préférentiel sur tranche transversale » n'est pas documenté dans l'Aurignacien (Le Brun-Ricalens 2005 ; Pesesse et Michel 2006), le Gravettien (Klaric 2003 ; Pesesse 2003 et 2008 ; Foucher 2004 ; Simonet 2004 ; Guillermin 
2006 ), ou même le Solutréen (Aubry et al. 1998 et 2004 ; Renard et Geneste 2006 ; Renard 2008 et soumis). Par ailleurs, sur plus d'une vingtaine de gisements comportant ce type de nucléus, seulement deux d'entre eux ont été, sur le seul indice de laminarité de l'industrie, attribués au Magdalénien moyen («Le Signal », fig. 15, nº 2 et KervazoDuchadeau 1982 ; «Guimberteau », Lenoir 1987). En revanche, l'ensemble des autres gisements, dont aucun ne présente un profil similaire à celui de la station éponyme ${ }^{11}$, a pu être rapporté à une phase ancienne du Magdalénien (sensu Bordes, cf. supra), et ceci soit par l'allure générale de l'industrie, soit grâce à la présence de certains « fossiles directeurs » tels que les raclettes (Lenoir 1987). Cependant, le contexte de récolte de la plupart de ces assemblages laisse planer le doute, la moitié d'entre eux correspondant à des ramassages de surface ne garantissant pas l'homogénéité du matériel. Cet état de fait, confronté aux données acquises ces dernières années sur les industries lithiques de la fin du Dernier Maximum Glaciaire montrant une diversification des schémas opératoires de production lamellaire, nous amène à appréhender une nouvelle fois la question de l'attribution chronoculturelle des industries à " pièces de La Bertonne » : "fossile directeur » (Lenoir 1987) probable du Magdalénien ancien... mais lequel ?

\section{La piste magdalénienne à l'épreuve des données actuelles}

39 Jusque-là documentées essentiellement dans les Cantabres et considérées dans cette zone géographique comme les premiers témoignages de la culture magdalénienne (Utrilla 1981, 1996; Cazals 2000), certaines industries découvertes sur le territoire français ont permis la clarification du cadre chrono-culturel post-solutréen. Ainsi, le Magdalénien inférieur, caractérisé par des assemblages riches en nucléus carénés et microlamelles à dosest donc venu, d'après les données stratigraphiques et radiométriques disponibles, s'intercaler entre le Badegoulien récent (ex Magdalénien I) et le Magdalénien moyen. Reconnu en Gironde (Cousté 1951 ; Lenoir et al. 1991), dans la Vienne (Primault et al. 2007), dans le Lot (Langlais et al. 2007a), dans le Tarn-et-Garonne (Millet-Conte 1994 et 1995 ; Ladier 2000 ; Langlais et al. 2007b) en passant par le Gard (Bazile et al. 1989), l'Hérault (Philippe et Bazile 2000), le versant nord-pyrénéen (Pétillon et al. 2008) et, très certainement, l'Yonne (Le Brun-Ricalens et Brou 2003), ce technocomplexe semble actuellement couvrir une bonne partie du territoire paléolithique français (Langlais 2007). Cette reconnaissance tardive nous amène actuellement à poser un regard critique non seulement sur certains assemblages dits "aurignacoïdes», mais également sur la variabilité fréquemment soulignée du Badegoulien $^{12}$. De fait, en raison de caractères techno-économiques qui, bien que distincts, laissent entrevoir une communauté de style (Ducasse et Langlais 2007), Badegoulien et Magdalénien inférieur ont longtemps été associés (voire "mélangés ») au sein du Magdalénien ancien sensu lato. Dès lors, la critique taphonomique de ce regroupement permet de s'interroger sur certaines attributions: si nous possédons actuellement une bonne image des productions lithiques de ces deux entités nous autorisant à les distinguer, les assemblages comme celui de Seyresse ou de La Bertonne continuent de semer le doute (Cretin et al. 2007 - p. 731).

La présence d'objets morphologiquement proches des " pièces de La Bertonne » au sein d'industries attribuées au Magdalénien inférieur a été signalée à plusieurs reprises (Soriano 2004 ; Langlais 2007). Il s'agit notamment des "nucléus grattoir/burin » décrits à Thèmes (Le Brun-Ricalens et Brou 2003), de certains grattoirs carénés « à front inverse » de Saint-Germain-la-Rivière (Lenoir et al. 1991- p. 249) ou de quelques burins 
« carénoïdes » de Gandil (Millet-Conte 1995 ; Ladier 2000 -p. 193). Toutes ces pièces correspondent effectivement à un ensemble cohérent, dont nous allons voir que s'il peut, par certains aspects morphologiques, être rapproché des nucléus décrits à Seyresse, il doit en être distingué sur de nombreux autres.

41 Cette modalité de débitage, dite « sur front ventral déjeté » (fig. 12, n 1 à 3 ; Langlais 2007) correspond à l'exploitation semi-transversale d'un support souvent allongé (lame ou éclat laminaire). L'ouverture de la table, si elle n'est jamais effectuée par le biais d'une "troncature " inverse, peut être réalisée soit à l'aide d'une fracturation volontaire du support, soit par le recours à un enlèvement «burinant »; ces opérations étant réalisées à partir d'un plan de frappe constitué par un pan de la face supérieure. Cependant, le rôle de l'arête ainsi créée (ou préexistante dans le cas de l'exploitation d'une extrémité légèrement rebroussée) stigmatise la différence entre ce schéma opératoire et le « débitage plan préférentiel sur tranche transversale » documenté à Seyresse : si dans ce dernier cas, la morphologie de l'arête conditionne directement la qualité du (ou des) support(s) recherché(s), dans le second, elle permet l'amorce du débitage destiné à se développer en récurrence dans l'épaisseur de la matrice. Il ne s'agit donc pas d'un débitage « sur tranche » exploitant la face étroite du support, mais d'une production "sur front" investissant la face large de celui-ci de manière subtransversale ${ }^{13}$. À l'état d'abandon, la plupart de ces nucléus arborent un front de débitage tout à fait semblable à certains des "grattoirs " carénés abondants dans ces industries (fig. $12, \mathrm{n}^{\circ} 4$ et 5 ; nucléus «sur front dorsal»), soulignant l'existence d'une pluralité de modalités, mises en œuvre afin de répondre à un objectif unique: la microlamelle torse à dos dextre et tranchant convexe. De fait, et à l'instar de M. Lenoir qui notait l'absence de "pièce de La Bertonne typique " à Saint-Germain-la-Rivière (Lenoir et al. 1991, op. cit.), il semble bien que nous ayons affaire à deux schémas opératoires différents sous-tendus par des objectifs distincts ${ }^{14}$. À titre d'exemple, le réexamen de la série de « la Cave à endives » dans le Lot (fig. $15, \mathrm{n}^{\circ} 22$ ), attribuée à un Magdalénien ancien ou moyen et au sein duquel étaient signalées deux « pièces de La Bertonne » (Raux et Piel-Desruisseaux 1997), a montré qu'une partie de ce matériel devait plus vraisemblablement être rapprochée du Magdalénien inférieur, ces pièces particulières correspondant finalement à des nucléus « sur front ventral déjeté $»^{15}$. 
Figure 12 - Quelles affinités technologiques pour les " pièces de la Bertonne »? Figure 12 - What are the technological affinities of the "pièces de la Bertonne »?

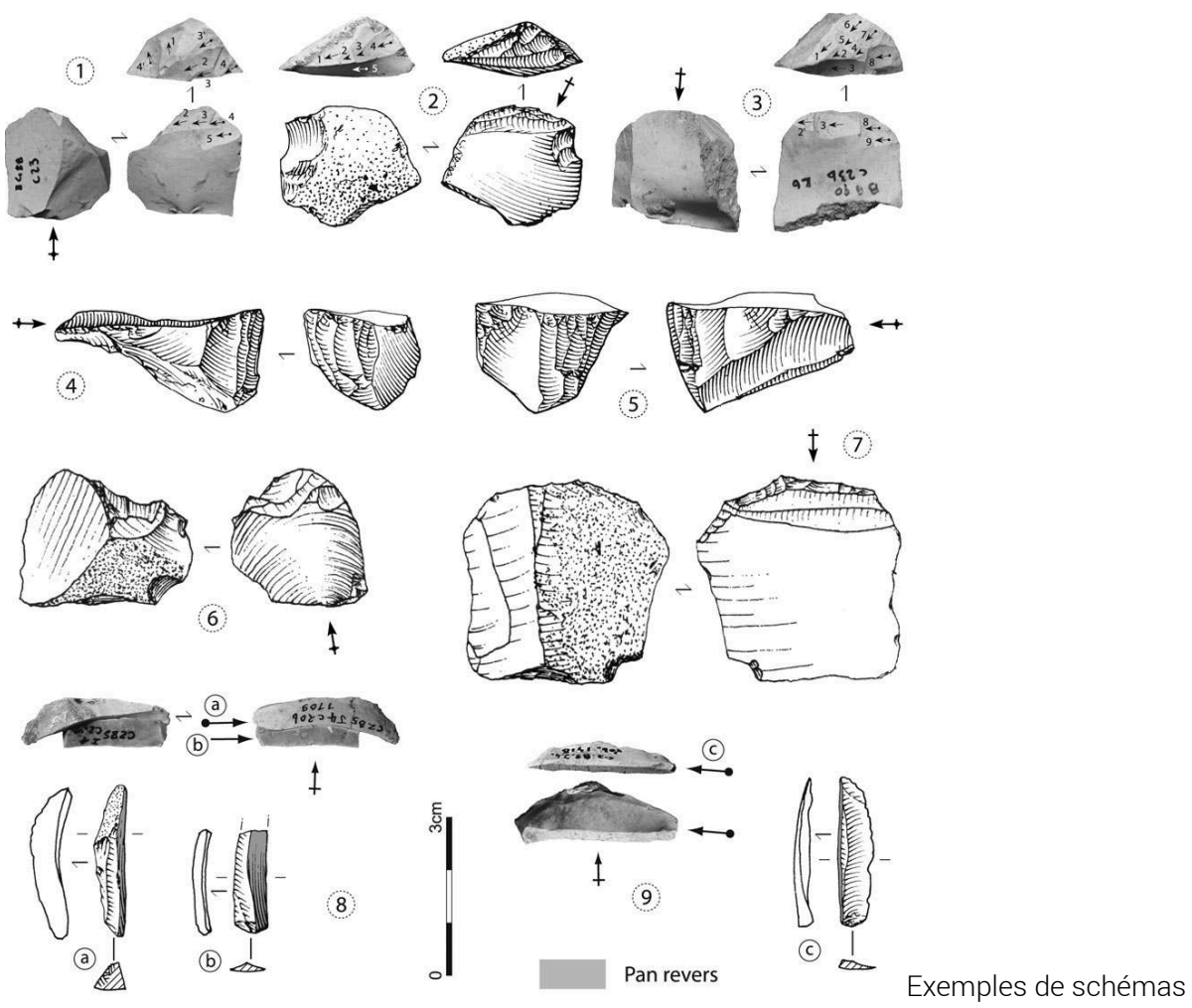

opératoires lamellaires du Magdalénien inférieur ( $n^{\circ} 1$ à 3 : débitage "sur front ventral déjeté »; $n^{\circ} 4$ et 5 : nucléus «sur front dorsal »-Gandil, dessins M. Jarry) et du Badegoulien ( $n^{\circ} 8$ et 9 : remontages de quelques séquences de débitage du type « préférentiel débordant »- Le Cuzoul de Vers, dessins $\mathrm{M}$. Jarry). Association (réelle ?) de nucléus « sur front ventral déjeté » $\left(n^{\circ} 6\right)$ et d’un débitage « plan préférentiel sur tranche transversale » ( $\left.n^{\circ} 7\right)$ à la Bombetterie (d'après Demars 1973).

Examples of bladelet operating schemes of lower Magdalenian ( $n^{\circ} 7$ to 3 : "sur front ventral déjeté » debitage ; $n^{\circ} 4$ and 5 : " sur front dorsal » cores - Gandil, drawings by M. Jarry) and Badegoulien ( $n^{\circ} 8$ and 9 : refits of some debitage sequences of "préférentiel débordant » type - Le Cuzoul de Vers, drawings by $M$. Jarry). (Real ?) Association of "sur front ventral déjeté » cores $\left(n^{\circ} 6\right)$ and "plan préférentiel sur tranche transversale » debitage $\left(n^{\circ} 7\right)$ in La Bombetterie (According to Demars 1973). 
Figure 13 - Sur le caractère badegoulien de l'industrie de Seyresse (Landes) : exemple de " burins à encoches » exploités selon un débitage "préférentiel débordant ".

Figure 13 - About the badegoulian feature of the assemblage of Seyresse (Landes) : examples of "notched burins » (" préférentiel débordant » debitage type).

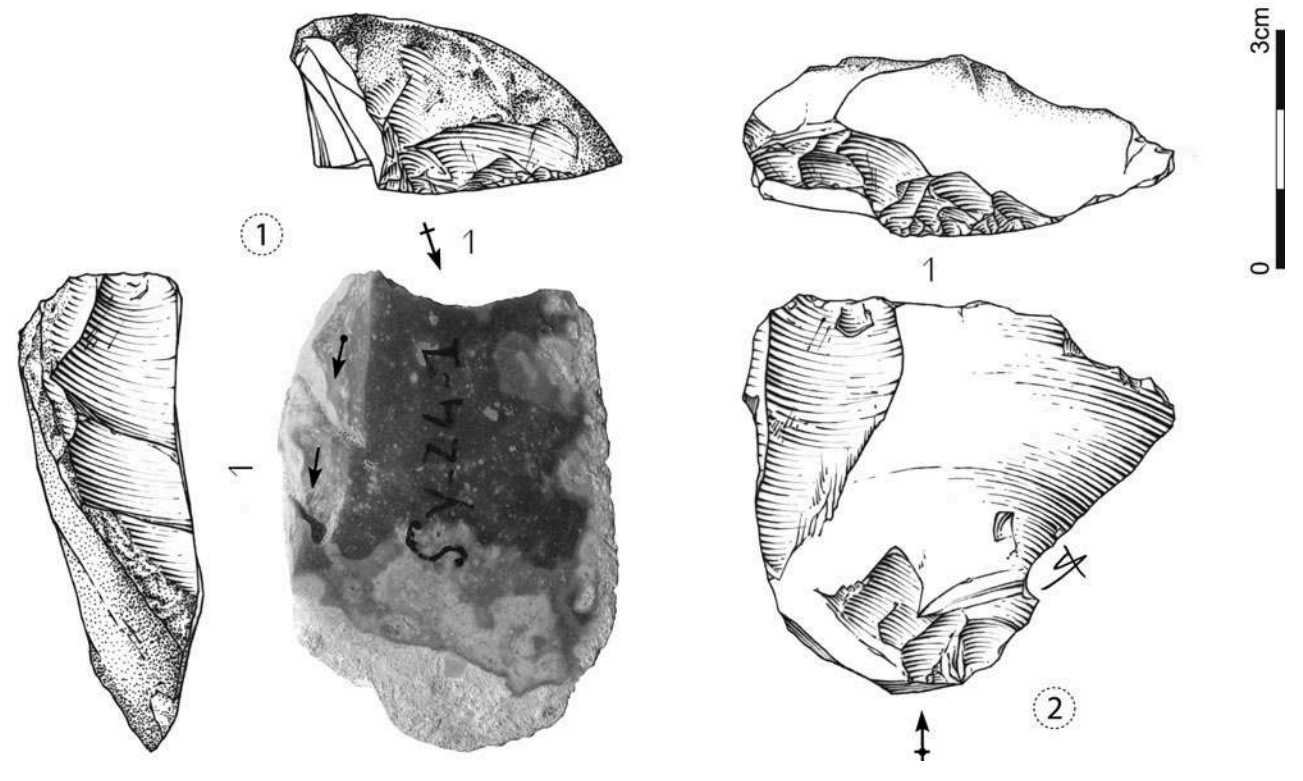

(dessins et clichés S. Ducasse).

Drawings and photos S. Ducasse. 
Figure 14 - Comparaisons avec les industries badegouliennes de Cabannes (Landes) et Lassac (Aude).

Figure 14 - Comparisons with the badegoulian industries of Cabannes (Landes) and Lassac (Aude).
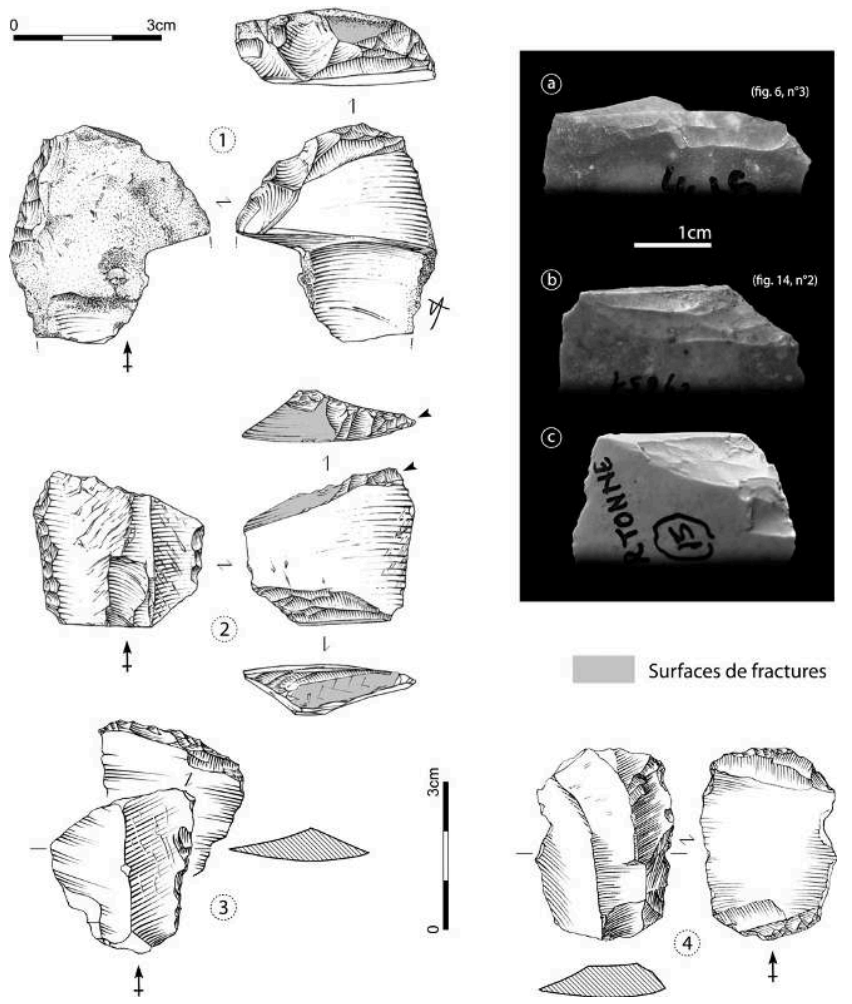

$n^{\circ} 1$ - débitage « préférentiel débordant » glissant vers une gestion proche des pièces de Seyresse (troncature inverse partielle ; variabilité du «burin transversal sur encoche »- Cabannes, dessin S. Ducasse) $; n^{\circ} 2$ : mésial de lame large exploité selon le schéma décrit à Seyresse (« pièce de la Bertonne »-Cabannes, dessin S. Ducasse); de «a » à « $C$ » : comparaison de la morphologie des tables (débitage par pression? a - Seyresse, b - Cabannes, c - La Bertonne) ; n 3 et 4 : débitage " plan préférentiel sur tranche transversale » issu de l'industrie de Lassac (ramassages Durand, d'après Sacchi 1986).

$n^{\circ} 7$ - «préférentiel débordant » debitage sliding towards an exploitation close to the pieces of Seyresse (partial inverse truncation, variability of the «transverse noched burin »-Cabannes, drawing S. Ducasse) $n^{\circ} 2$ : «Pièce de la Bertonne » on a mésial of wide blade (Cabannes, drawing S. Ducasse); from «a » to " $C$ » : comparison of the morphology of the flaked surfaces (pressure debitage? a - Seyresse, $b$ - Cabannes, $c$ La Bertonne) ; $n^{\circ} 4$ et 5 : " plan préférentiel sur tranche transversale » debitage from the industry of Lassac (Durand's collection, Sacchi 1986). 
Figure 15 - « Pièces de La Bertonne » versus "pièces d'Orville » : localisation des principaux assemblages où sont documentées ces modalités de production.

Figure 15 - "Pièces de la Bertonne " versus " pièces d'Orville » : location of the main assemblages where these modalities of production are documented.

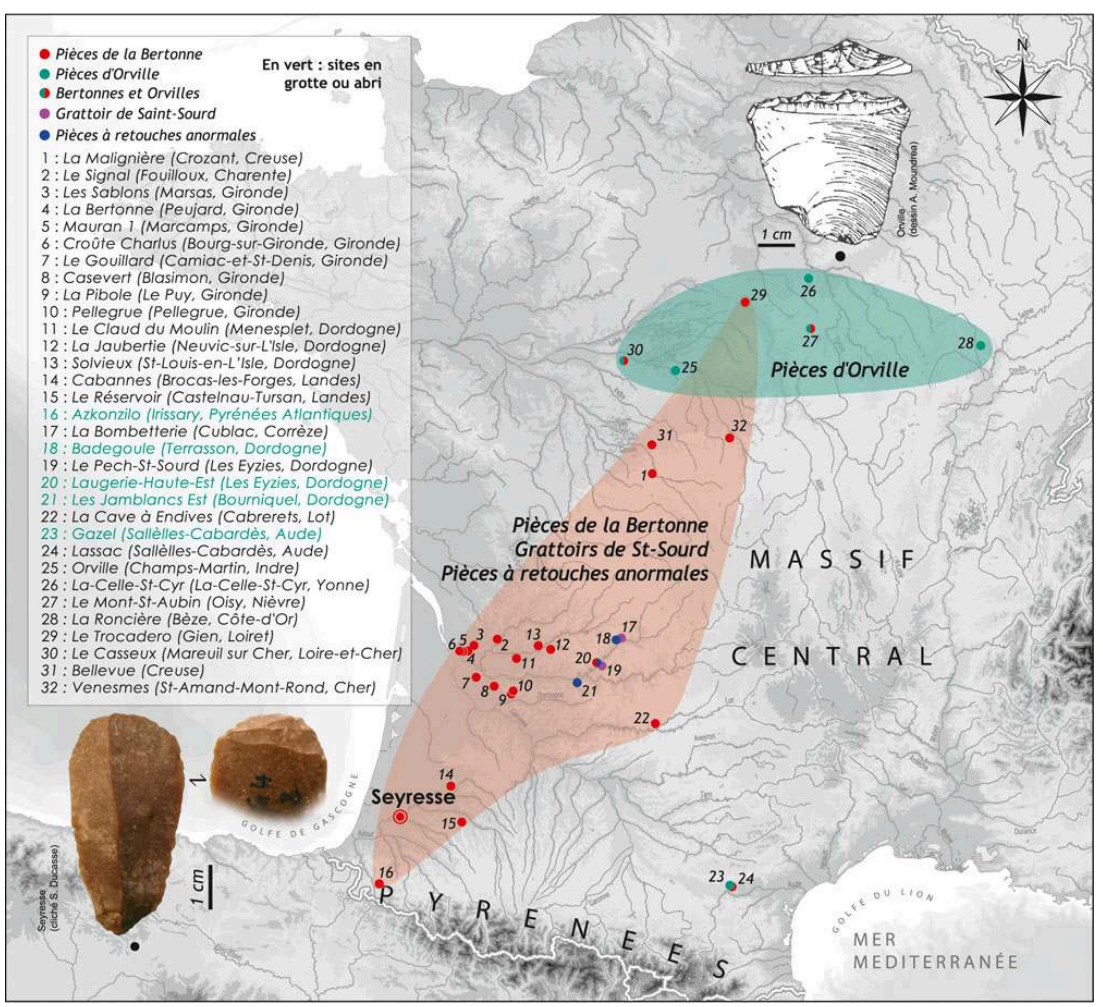

(d'après Bodu et Debout 2004 ; Cretin 2000 ; Dachary 2002 ; Demars 1985 ; Demars et Laurent 1989 ; Ducasse 2004 ; Ferullo et Vigier 1997 ; Fourloubey et al. 2006 ; Ortega et al. 2005 ; Langlais 2007 ; Lenoir 1983, 1987 ; Leysalles et Noone 1949 ; Merlet 2005 ; Parisot 1995 ; Perlès 1977, 1982 ; Raux et Piel-Desruisseaux 1997 ; Sackett 1999 ; Soriano et Lechenet 2000 ; Trotignon 1985 et 1993 ; Renard 2002 ; Valentin 1995)

La distinction entre ces nucléus et le «débitage plan préférentiel sur tranche transversale" semble manifeste à la Bombetterie (fig. 12, $n^{\circ} 6$ et 7). En effet, la consultation des planches publiées nous amène à considérer leur coexistence au sein de cette industrie, par ailleurs recueillie en surface par A. Cheynier autour des années 1950, l'attribuant alors à un Aurignacien final (Cheynier 1956). Si cette association permet d'imaginer l'appartenance de ces « grattoirs de Saint-Sourd » (Demars 1973) au Magdalénien inférieur (auquel, d'après les publications, une partie du matériel semble pouvoir correspondre), les conditions de collecte du matériel (ramassages sur plusieurs locus) ainsi que le caractère isolé de ce "couple technologique» incitent à rester prudent. En attendant une analyse technologique complète - incontournable - de ce matériel, nous privilégierons, en terme de tendance, une distinction chronoculturelle entre ces deux modalités considérant, nous allons le voir, que c'est avec le Badegoulien que les liens technologiques semblent les plus étroits ${ }^{16}$.

\section{Une modalité d'essence badegoulienne?}

43 En effet, c'est avec les «burins transversaux sur encoche » du Badegoulien que nous avons pu effectuer les comparaisons les plus intéressantes. Cette modalité qui, bien que pressentie par un certain nombre de chercheurs n'a été décrite dans sa dynamique qu'au début des années 2000 à partir des industries de Oisy (Bodu et Senée 2001 ; Bodu 
2003), constitue une «formule» (Cazals 2000) permettant de répondre à des objectifs divers, notamment lamellaires (Cretin et al. 2007 ; Bodu et al. 2007 ; Ducasse et Langlais 2007). Reconnue par la suite dans de nombreux autres gisements (Chehmana 2004 et thèse en cours; Chehmana et al. 2007 ; Ducasse 2004 et thèse en cours) elle correspond, dans la plupart des cas, au schéma "phare » de ces assemblages, quasi-exclusif lorsqu'il s'agit de produire des supports lamellaires (d'autres modalités sont documentées, mais en proportions souvent infimes). Largement décrite par ailleurs, nous n'en rappellerons que les traits essentiels nécessaires aux comparaisons présentées ici.

Cette "formule » consiste donc, dans sa version lamellaire, en l'exploitation de la tranche transversale d'un éclat épais plus ou moins allongé, afin d'en extraire un nombre relativement limité de supports par séquences. L'aménagement de la table s'effectue le plus souvent par le biais d'un enlèvement «burinant» qui vient, de manière tout à fait similaire aux pièces décrites à Seyresse, « conformer » la table par la création d'une arête transversale constituée par la rencontre de cet enlèvement et de la face inférieure de l'éclat. Cette nervure est alors mise à profit, à partir d'un plan de frappe aménagé latéralement («encoche»), lors de l'extraction lamellaire dont le « rythme " peut être considéré comme " préférentiel ». En effet, la récurrence de ces enlèvements est extrêmement rare (une à deux lamelles par séquence), la poursuite du débitage étant rythmée par des réaménagements tabulaires conséquents (enlèvements «burinant» épais). Ce schéma opératoire est donc guidé par la recherche de fines lamelles rectilignes dont la plus grande part porte un résidu de la face inférieure de la matrice (débordement); pan revers plus ou moins abrupt (fig. $12, \mathrm{n}^{\circ} 8$ et 9 ). Ce " débitage préférentiel débordant sur tranche transversale » badegoulien (Ducasse et Langlais 2007; Ducasse et Lelouvier soumis) présente, ainsi décrit, de nombreux points communs avec le « débitage plan préférentiel sur tranche transversale » de Seyresse. Bien qu'il existe des distinctions au niveau des procédés mis en œuvre (façonnage d'une troncature inverse, type de plan de frappe), justifiant de probables différences dans la morphologie des produits recherchés (caractère "plan " accru à Seyresse peut-être lié à la recherche de supports plus graciles), la structure générale de ces débitages leur confère un air de famille certain.

Leur proximité conceptuelle ainsi que, dans les deux cas, leur mise en œuvre privilégiée (voire quasi-exclusive) au sein des assemblages qui les abritent, incitent à considérer un véritable rapprochement culturel entre les deux, confirmant ainsi - en les précisant les premières approches typologiques (Lenoir 1987). Cette relation, qui revient donc à associer (en tout cas conceptuellement) industries à raclettes et industries à " pièces de La Bertonne ", trouve des arguments supplémentaires au sein de l'industrie de Seyresse elle-même, ainsi que dans son environnement archéologique proche. En effet, s'il n'y existe aucun nucléus à lamelles « classique » (prismatique ou pyramidal), l'assemblage de Seyresse compte trois éclats dont la tranche montre clairement la recherche de supports allongés. Or, deux d'entre eux semblent pouvoir être rapprochés des «burins transversaux sur encoche » badegouliens, l'orientation de leur table (longitudinale) mise à part (fig. 13). En même temps qu'elles illustrent la variabilité de ce schéma (Ducasse, soumis), ces deux pièces permettent de documenter une intention probablement distincte du « débitage plan préférentiel sur tranche transversale » (les "Bertonnes »), les supports produits ne supportant que difficilement la comparaison.

Par ailleurs, les travaux de J.-C. Merlet (Merlet 2005), s'ils ont offert une meilleure connaissance des occupations badegouliennes régionales, ont en outre permis la mise 
au jour d'indices tout aussi intéressants. Ainsi, au-delà du signalement de nouveaux gisements de surface documentant cette association "raclettes / pièces de la Bertonne » (Le Réservoir ; fig. 15, $\mathrm{n}^{\circ} 15$ ), les données issues de la fouille du gisement de plein air de Cabannes nous semblent décisives pour notre propos (fig. 1 et fig. $15, \mathrm{n}^{\circ} 14$; Gellibert et Merlet 2001). La mise en évidence de ce «débitage plan préférentiel sur tranche transversale" au sein d'un assemblage homogène, marqué par la présence massive de raclettes (plus de 1000 exemplaires) et d'un abondant débitage laminaire et lamellaire (Ducasse 2004), a constitué pour nous un argument solide de rapprochement malgré le caractère unique de la pièce (fig. $14, n^{\circ} 2$ ). Cette dernière, réalisée sur un fragment mésial de lame large, présente en effet à elle seule l'ensemble des caractères documentés sur les pièces de Seyresse :

1. chacune des tables montre les résidus d'une fracturation volontaire, la table distale ayant quant à elle été régularisée par le biais d'une « troncature » inverse partielle ;

2. les plans de frappe sont indifféremment constitués par un pan de la face supérieure (table proximale) ou par une reprise directe du bord (table distale);

3. les lamelles recherchées sont extraites aux dépens de l'arête située à la jonction fracture/ face inférieure de la lame, la table proximale présentant un plus grand nombre de tentatives. Enfin, cette pièce offre un dernier intérêt : si la table proximale ne semble pas pouvoir être distinguée des exemplaires issus du gisement éponyme (fig. 14, b et c), la seconde, observée seule, pourrait tout à fait être interprétée comme une "pièce d'Orville " ${ }^{17} \ldots$

\section{4 - Implications et discussion}

Le regard technologique porté sur la série de Seyresse ouvre un certain nombre de perspectives pour la compréhension de ces industries particulières. En effet, confrontée aux données les plus récentes, l'analyse de cet assemblage permet de rediscuter de l'attribution culturelle du phénomène «Bertonne " ainsi que de ses relations avec ses « cousins » d'Orville et du Pech Saint-Sourd.

\section{1 - Quels Badegouliens?}

Les liens évoqués entre l'industrie de Seyresse et les assemblages attribués au Badegoulien, non plus seulement sur des critères typologiques (Lenoir 1987; Merlet 2005), mais avant tout par le biais de comparaisons technologiques, s'insèrent dans une réflexion sur la structuration à la fois diachronique et synchronique de ce technocomplexe. Remise en cause dans la moitié nord de la France (Bodu et al. 2007), la structuration interne du Badegoulien telle qu'elle a pu être perçue à partir d'un certain nombre de stratigraphies de qualité variable (Badegoule, Laugerie-Haute, Fritsch, Cassegros, Casserole, le Cuzoul de Vers, etc.) privilégie un découpage chronologique basé en grande partie sur les critères de présence ou d'absence de certains éléments jugés caractéristiques. Ainsi se succèdent Badegoulien ancien et Badegoulien récent, distingués sur les proportions relatives du couple " raclettes / burins transversaux " marquées par une évolution inverse (Cheynier 1939; Bordes 1958; Le Tensorer 1981 ; Trotignon et al. 1984; Clottes et Giraud 1989; Detrain et al. 1991). Si les données récentes acquises au Cuzoul de Vers ne nous permettent pas de contredire cette succession (Ducasse et Lelouvier, soumis), elles conduisent par contre à en réévaluer la dynamique, bien plus complexe que ces seules questions de proportions (qui, par 
ailleurs, ne se vérifient pas systématiquement, notamment concernant les «burins transversaux »). Mais ce qu'il faut souligner, c'est l'inadéquation de ces cadres avec un assemblage comme celui de Seyresse. À l'inverse du Badegoulien ancien qui n'est jusqu'à présent documenté clairement qu'en stratigraphie, les gisements à nombreuses «pièces de La Bertonne » ne se rencontrent exclusivement qu'en plein air (fig. 12), de sorte qu'il a été jusqu'ici difficile de leur assigner une place précise au sein de cette structuration. Il apparait en outre que les rares occurrences de la présence de ce type de nucléus en contexte stratigraphique sont à mettre en relation avec les seules occupations badegouliennes de Laugerie-Haute (Demars et Laurent 1989 ; Cretin 2000), de Badegoule (Cretin 2000 ; Bodu 2005 et obs. pers. ${ }^{18}$ ) ou des Jamblancs (Cretin 1993), et ne concernent qu'à peine $4 \%$ du total des pièces recensées. Cette dichotomie « sites à nombreuses Bertonnes et sans raclette/sites à nombreuses raclettes et très rares Bertonnes » incite à aborder une approche synchronique de ces assemblages par le biais d'une différentiation fonctionnelle. Une telle interprétation implique l'existence d'une complémentarité inter-sites qui semble malheureusement difficile à étayer sur la base des données recueillie à ce jour : si nous ne réfléchissons que sur une partie des manifestations techniques liées à ces occupations (absence d'industrie osseuse et de faune conservée), le sous-système abordé, en l'occurrence lithique, est lui-même le plus souvent incomplet, à l'image de Seyresse où les intentions économiques du schéma opératoire décrit dans cet article restent inconnus, faute de fraction fine...

\section{2 - Bertonnes versus Orvilles : un éternel débat?}

Finalement aussi délicate que celle de la Bertonne, l'attribution culturelle de l'industrie d'Orville pose un type de problème similaire : reliée au Magdalénien moyen, elle n'en réunit pourtant aucun marqueur lithique incontestable (si ce n'est l'existence de quelques nucléus de type «La Marche» - Parisot 1995). Ainsi, c'est une nouvelle fois la présence - sporadique - de « pièces d'Orville » dans des contextes mieux maîtrisés qui a permis d'asseoir cette hypothèse (Parisot 1995; Soriano et Lechenet 2000; Angevin 2008). Aujourd'hui est donc privilégiée une distinction non seulement technologique, mais aussi culturelle et géographique, entre "pièces de la Bertonne" et "pièces d'Orville»; la répartition des gisements qui renferment ces deux types d'objets montrant de façon générale une bipartition nord/sud attendue (fig. 15). Nous pensons cependant que les données apportées ici permettent une nouvelle fois d'aborder la question épineuse du rapport entre ces deux schémas opératoires dont la relation nous semble moins « formelle que conceptuelle »(Perlès 1982 - p. 131).

Certains exemplaires de Seyresse (comme la pièce décrite à Cabannes; fig. 14, $\mathrm{n}^{\circ} 2$ ) soulignent en effet l'impossibilité fréquente d'une distinction entre "Orvilles» et «Bertonne » (fig. 8, $\mathrm{n}^{\circ} 1$ et 2 ), de sorte que nous aurions bien pu - à tort - considérer cet assemblage comme associant les deux modalités. Au contraire, l'emploi d'un vocabulaire descriptif neutre lors de l'analyse a permis de dépasser la barrière psychologique imposée par l'utilisation de termes éponymes. À l'heure actuelle, la seule et unique différence qu'il serait possible d'évoquer pour les distinguer concernerait, tout comme l'ont fait $\mathrm{M}$. Lenoir et $\mathrm{C}$. Perlès, la multiplicité des enlèvements observables sur les "pièces de la Bertonne » (au contraire des nucléus d'Orville qui n'en comptent généralement qu'un seul). Il est intéressant, à cet égard, de remarquer que si les "pièces d'Orville » ont été assez rapidement distinguées des "pièces de la Bertonne " sur cet argument, elles ont paradoxalement été rapprochées des "grattoirs de Saint- 
Sourd " (Perlès 1977 - p. 141) alors même que ces derniers peuvent présenter jusqu'à cinq négatifs lamellaires consécutifs (Leysalles et Noone 1949). En définitive, cet argument ne nous semble pas assez solide pour permettre une distinction nette de la gestion de ces productions, considérant au contraire qu'il s'agit d'une seule et même modalité de débitage lamellaire exprimée dans sa variabilité. La rareté des gisements connus pour la présence de "pièces d'Orville» qui, le site éponyme excepté, en possèdent par ailleurs peu d'exemplaires, contraste avec le nombre relativement important de sites à "pièces de la Bertonne » (présents pour leur part sur un espace plus étendu). Ceci, ajouté au fait qu'il existe, aux marges de la zone de présence des "Orvilles» (voire en son sein), des industries à «pièces de la Bertonne " (Trotignon 1985 et 1993 ; Valentin 1995 ; Chehmana, thèse en cours) nous amène à nous interroger à la fois sur la pertinence de cette distinction et sur le caractère référant du site d'Orville. Sans pour autant franchir le pas d'une attribution badegoulienne de ce dernier, nous pensons que cette hypothèse mérite largement d'être discutée, même si nous n'oublions pas que, pensé dans sa variabilité, ce schéma opératoire peut correspondre à une modalité transculturelle dont le succès a pu varier avec le temps et les comportements économiques. Nous ne doutons pas que les recherches actuelles menées sur les gisements de la moitié nord de la France viendront très rapidement alimenter un débat déjà largement ouvert par la découverte de l'industrie de Oisy (Bodu et Senée 2001).

51 Concernant le sud de la France, ces propositions mériteront donc d'être évaluées à l'avenir sur une base double: une réévaluation techno-économique préalable des principaux assemblages à « Bertonnes » appuyée, nous ne pouvons que le souhaiter, par l'analyse d'un matériel plus complet, aussi bien dans la nature des vestiges recueillis (lithiques, osseux, etc.) que dans leur représentativité ${ }^{19}$.

\section{Remerciements}

Les auteurs tiennent à remercier vivement C. Normand, D. Barraud, J.-C. Merlet, M. Lenoir, E. Ladier, J. Clottes, J.-P. Giraud et D. Sacchi pour l'accès au matériel des différentes séries mobilisées pour cet article. Enfin, il nous est agréable de remercier $C$. Fourloubey pour cette journée de discussion passée autour des pièces du Casseux ainsi que C. Cretin, J.J. Cleyet-Merle, F. Bon et L. Brou pour leurs conseils et relectures.

\section{BIBLIOGRAPHIE}

ALIX P., PELEGRIN J. et DELOGE H. 1995 - Un débitage original de lamelles par pression au Magdalénien du Rocher-de-la-Caille (Loire, France), PALEO,7, p. 187-199.

ANGEVIN R. 2008 - Enquête sur la variabilité des systèmes de production lamellaire au sein de la séquence magdalénienne du Massif Central et de ses marges. Apports des industries lithiques de la Corne-de-Rollay (Couleuvre, Allier), d'Enval 1 (Vic-le-Comte, Puy-de-Dôme) et du Blot (Cerzat, Haute-Loire). Mémoire de Master 2 de l'université de Paris I, $134 \mathrm{p}$. 
ARAMBOUROU R. 1970 - Un campement protosolutréen à Seyresse (Landes). Bull. Soc. de Borda, 337, p. 3-11.

AUBRY T., WALTER B., ALMEIDA M., LIARD M., NEVES M.-J. 2004 - Approche fonctionnelle des sites dit d'atelier : l'exemple des occupations solutréennes et badegouliennes des Maitreaux (Indre-et-Loire, France). In : XX⿳o Congrès Préhistorique de France. "Approches fonctionnelles en Préhistoire". Nanterre 24-26 novembre 2000. p. 249-264.

AUBRY T., WALTER B., ROBIN E., PLISSON H., BENHABDELHADI M. 1998 - Le site solutréen de plein air des Maîtreaux (Bossay-sur-Claise, Indre-et-Loire) : un faciès original de production lithique. PALEO, décembre 1998, no 10, p. 163-184.

BAZILE F., GUILLERAULT Ph. et MONNET C. 1989 - L'habitat paléolithique supérieur de plein air de Fontgrasse, Travaux 1983-1987, Gallia Préhistoire, t. 31, p. 65-92.

BODU P. 2003 - Le Badegoulien de la nièvre est un Badegoulien à lamelles à dos ! Nouvelles découvertes effectuées sur le site de Oisy dans la Nièvre. In : P. BODU et S. SORIANO coord., Le Paléolithique supérieur ancien au centre et au sud du Bassin parisien « des systèmes techniques aux comportement, rapport de PCR, Programme P4, Région Centre-Nord, p. 79-84.

BODU P. 2005 - État des connaissances sur la culture matérielle badegoulienne dans le nord de la France. In P. Bodu et L. Chehmana dir., Le Paléolithique supérieur ancien au centre et au sud du Bassin parisien, "Des systèmes techniques aux comportements », rapport de PCR dans le cadre du programme P4, année 2005, région Centre-Nord, p. 74-91.

BODU P., CHEHMANA L. et DEBOUT G. 2007 - Le Badegoulien de la moitié nord de la France. Un état des connaissances, Bulletin de la Société préhistorique française, t. 104, nº 4, p. 661-679.

BODU P. et DEBOUT G. 2004 - Le gisement badegoulien du Mont-St-Aubin, Oisy (Nièvre), second rapport préliminaire de fouille programmée (autorisation tri-annuelle 2003-2005), Avril 2004, $80 \mathrm{p}$.

BODU P. et SENEE A. 2001 - Le gisement « badegoulien » du Mont-saint-Aubin à Oisy (Nièvre). Archéologie en Haut-Nivernais-Vaux d'Yonne Archéologie 19, p. 3-59.

BON F., CHAUVAUD D., DARTIGUEPEYROU S., GARDERE Ph., MENSAN R. 1996 - La caractérisation du silex de Chalosse, Antiquités Nationales, 28, p. 33-38.

BORDES F. 1958 - Nouvelles fouilles à Laugerie-Haute Est. Premiers résultats. L'Anthropologie, LXII, p. 205-244.

BRACCO J.P., MORALA A., CAZALS N., CRETIN C. et FERRULLO O. 2003 - Peut-on parler de débitage discoïde au Magdalénien ancien/Badegoulien? Présentation d'un schéma opératoire de production d'éclats courts normalisés. In : M. PERESANI éd., Discoid lithic technology Advances and implications, BAR 1120, p. 83-115.

CASTEL J.C., CHAUVIERE F., LHOMME X. et CAMUS H. 2006 - Un nouveau gisement du Paléolithique supérieur récent : le Petit Cloup Barrat (Cabrerets, Lot, France). Bulletin de la Société préhistorique française, t. 103, $\mathrm{n}^{\circ}$ 2, p. 263-273.

CAZALS N. 2000 - Constantes et variations des traits techniques et économiques entre le Magdalénien inférieur et moyen : analyse des productions lithiques du Nord de la péninsule ibérique, Thèse de doctorat de l'université de Paris I - Panthéon-Sorbonne, ex. multigraphié.

CHALARD P., DUCASSE S., BON F., BRUXELLES L., TEYSSANDIER N., RENARD C., GARDERE P., GUILLERMIN P., LACOMBE S., LANGLAIS M., MENSAN R., NORMAND C., SIMONNET R. et TARRINO A. sous presse - Diffusion et exploitation d'un traceur lithologique au cours du Paléolithique 
supérieur dans le sud de la France : l'exemple du type Chalosse, In : A. BURKE dir., Quarries : where it al began, Actes du congrès international de la S.A.A., Porto Rico, 2006.

CHEHMANA L. 2004 - Enquète sur l'identité du Badegoulien et ses origines possibles. Apport de l'étude des industries lithiques badegoulienne de Oisy (Nièvre) et solutréennes de Saint-Sulpice-de-Favière (Essonne) et de Fressignes (Indre), Mémoire de DEA de l'université de Paris I, 55 p.

CHEHMANA L., LIARD M., BODU P. 2007 - De nouveaux arguments pour un rattachement au Badegoulien des séries dites « à grands burins transversaux » de la vallée de la Claise. Bulletin de la Société préhistorique française, t. 104, n 4, p. 681-697.

CHEYNIER A. 1930 - Un outil magdalénien nouveau en silex à Badegoule : la raclette. Bulletin de la Société préhistorique française, t. 27, p. 483-488.

CHEYNIER A. 1939 - Le Magdalénien primitif de Badegoule. Niveaux à raclettes. Bulletin de la Société préhistorique française, t. 34, n 9, p. 354-396.

CHEYNIER A. 1956 - La Bombetterie, station aurignacienne de plein air à Cublac (Corrèze). In : Libro homenaje al conde de la Vega del Sella, Diputación provincial de Asturias, SIA, p. 95-106.

CLOTTES J. et GIRAUD J.-P. 1989 - Le gisement préhistorique du Cuzoul (Vers, Lot). Quercy recherche, $1989, \mathrm{n}^{\circ}$ 65-66, pp. 82-91.

COUSTE R. 1951 - Gisement magdalénien des grottes de Jaurias, Bulletin de la Société préhistorique française, t. 48, p. 381-384.

CRETIN C. 1993 - Le Magdalénien inférieur de l'abri Ouest des Jamblancs. Étude techno-économique du matériel lithque. Mémoire de DEA de l'université de Paris I, 166 p.

CRETIN C. 2000 - Tradition et variabilité dans le comportement technique. Le cas du Badegoulien et du Magdalénien en Périgord. Thèse de Doctorat, Université de Paris I, ex. multigraphié, 451 p.

CRETIN C., FERULLO O., FOURLOUBEY Ch., LENOIR M. et MORALA A. 2007 - Le Badegoulien du nord de l'Aquitaine : de nouveaux moyens de lecture, Bulletin de la Société préhistorique française, t. $104, \mathrm{n}^{\circ} 4$, p. $715-734$.

DACHARY M. 2002 - Le Magdalénien des Pyrénées occidentales. Thèse de Doctorat, Université de Paris X, ex. multigraph, $300 \mathrm{p}$.

DALEAU F. 1910a - Silex à retouches anormales de la station de la Bertonne ou la Rousse, commune de Peujard, Gironde, Actes de la Société Archéologique de Bordeaux, t. 31.

DALEAU F. $1910 \mathrm{~b}$ - Encore les silex à retouches inverses, A. F. A. S., $39^{\text {ème }}$ session, Toulouse, notes et mémoires, t. II, p. 275-276.

DELOGE H. et DELOGE L. dir. 2003 - Le Rocher de la Caille. Un site magdalénien de plein air au Saut-duPerron (Saint-Jean/Saint-Maurice-sur-Loire, Loire). Mémoire de la société préhistorique française, $\mathrm{n}^{\circ} 31,252 \mathrm{p}$.

DEMARS P.-Y. 1973 - Le gisement aurignacien de la Bombetterie, commune de Cublac (Corrèze). Essai de comparaison morphologique avec l'outillage de type aurignacien de Chanlat et Bos del Ser, Bulletin de la Société préhistorique française, t. 70, p. 311-323.

DEMARS P.-Y. 1985 - La station de plein air magdalénienne de la Malignière, commune de Crozant (Creuse), Etudes Archéologiques, Crozant, Creuse, Documents d'Archéologie creusoise, 1, p. 27-39.

DEMARS P.-Y. et LAURENT P. 1989 - Types d'outils lithiques du Paléolithique supérieur. Cahiers du Quaternaire, 7. 
DETRAIN L., KERVAZO B., AUBRY Th., BOURGUIGNON L., GUADELLI J.-L., MARCON V., TEILLET Ph. 1991 - Agrandissement du Musée national de Préhistoire des Eyzies. Résultats préliminaires des fouilles de sauvetage. PALEO, $\mathrm{n}^{\circ}$ 3, p. 75-91.

DUCASSE S. 2004 - Produire des lames et des lamelles au Badegoulien : technologie et économie Analyse préliminaire d'ensembles lithiques du Badegoulien méridional. Mémoire de DEA, Université ToulouseLe Mirail, ex. multigraphié.

DUCASSE S. soumis - Badegoulien versus Magdalénien. I - le Badegoulien récent quercinois : état de la recherche, réflexions et questionnements. In : JARRY M., BRUGAL J.-Ph. et FERRIER C. dir., Modalités d'occupations et exploitation des milieux au Paléolithique dans le Sud-Ouest de la France: l'exemple du Quercy, Actes du colloque 67 du XVème congrès de l'UISPP, Lisbonne, Septembre 2006.

DUCASSE S. et LANGLAIS M. 2007 - Entre Badegoulien et Magdalénien, nos coeurs balancent... Approche critique des industries lithiques du Sud de la France et du Nord-Est espagnol entre 19.000 et 16.500 BP, Bulletin de la Société préhistorique française, t. 104, nº 4, p. 771-785.

DUCASSE S. et LELOUVIER L.-A. soumis - L'exploitation des matériaux siliceux durant le Badegoulien au Cuzoul de Vers : une première approche diachronique, In : J. CLOTTES et alii dir., Diversité et identité des groupes solutréens et badegouliens en Quercy : l'exemple de l'abri du Cuzoul de Vers, Lot.

FERULLO O. et VIGIER S. 1997 - Menesplet, «Le Claud du Moulin », DFS de sauvetage urgent, SRA Aquitaine, Novembre 1997, $164 \mathrm{p}$.

FOUCHER P. 2004 - Les industries lithiques du complexe Gravettien-Solutréen dans les Pyrénées. Technotypologie et circulation des matières premières de part et d'autre de l'axe Pyrénées/Cantabres, Thèse de doctorat de l'université de Toulouse II - Le Mirail, 3 vol. , 334 p.

FOURLOUBEY C. 1996 - Etude de la variabilité des industries post-solutréennes de transition vers le Magdalénien par l'analyse des travaux de taille du silex. L'exemple du Badegoulien et du Magdalénien ancien de plein air dans la moyenne vallée de l'Isle (Dordogne). Thèse de doctorat de l'Université de Bordeaux I. 1996, 250 p., 236 fig., 33 pl.

FOURLOUBEY C., BIDARD P., DELOZE V., GARAIZAR J.-R. 2006 - Le Casseux : sur les traces des premiers Magdalénien du Centre de la France. Rapport final d'opération de fouille archéologique préventive, Direction générale Centre, Ile de France, 224 p.

GELLIBERT B. et MERLET J.C. avec la coll. de FERRIER C. et LEGIGAN P. 2001 - Le gisement badegoulien de Cabannes (Brocas-les-Forges, Landes), Archéologie des Pyrénées occidentales et des Landes, t. 20, p. 81-104.

GUILLERMIN P. 2006 - Les Fieux : Une occupation gravettienne du causse quercinois, PALEO, $\mathrm{n}^{\circ} 18$, Décembre 2006, p. 69-94.

KERVAZO-DUCHADEAU C. 1982 - Recherches sur l'occupation paléolithique dans le bassin de la Dronne. Thèse de Doctorat en Géologie du Quaternaire et Préhistoire, Université de Bordeaux I, 939 p.

KLARIC L. 2003 - L'unité technique des industries à burins du Raysse dans leur contexte diachronique : réflexions sur la diversité culturelle au Gravettien à partir des données de la Picardie, d'Arcy-sur-Cure, de Brassempouy et du Cirque de la Patrie, Thèse de Doctorat, université de Paris I-Panthéon-Sorbonne, $426 \mathrm{p}$.

LADIER E. 2000 - Le Magdalénien ancien à lamelles à dos de l'abri Gandil à Bruniquel (Tarn-etGaronne) : étude préliminaire de l'industrie de la C.20, in G. PION dir., Le Paléolithique supérieur 
récent : nouvelles données sur le peuplement et l'environnement, Table-Ronde de Chambéry, 1999, Mémoires de la SPF, t. 28, p. 191-200.

LANGLAIS M. 2007 - Dynamiques culturelles des sociétés magdaléniennes dans leurs cadres environnementaux. Enquête sur 7000 ans d'évolution de leurs industries lithiques entre Rhône et Èbre. Thèse de Doctorat des Universités de Toulouse II - le Mirail et de Barcelone, ex. multigraphié.

LANGLAIS M., DUCASSE S., DAULNY L., MORALA A. et RENARD C. 2007a - Les vestiges lithiques en silex du Petit Cloup Barrat (campagne 2007). In : J.-C. Castel coord., Petit Cloup Barrat, Cabrerets, Lot. Rapport de fouille programmée, campagne 2007.

LANGLAIS M., LADIER E., CHALARD P., JARRY M. et LACRAMPE-CUYAUBERE F. 2007b - Aux origines du Magdalénien « classique » : les industries de la séquence inférieure de l'abri Gandil (Bruniquel, Tarn-et-Garonne), PALEO, 19, Décembre 2007, p. 341-366.

LE BRUN-RICALENS F. dir. 2005 - Productions lamellaires attribuées à l'Aurignacien, chaînes opératoires et perspectives technoculturelles. Actes du XIVe Congrès de l'IUSPP, Liège, 2001, Archéologiques 1 , $568 \mathrm{p}$.

LE BRUN-RICALENS F. et BROU L. 2003 - Burins carénés-nucléus à lamelles : identification d'une chaîne opératoire particulière à Thèmes (Yonne) et implications, Bulletin de la Société préhistorique française, t. 100, p. 67-83.

LENOIR M. 1976 - Étude technique et typologique des « pièces à retouches anormales » de la station de la Bertonne, commune de Peujard, Gironde, Bulletin de la Société préhistorique française, t. 73, p. $43-47$.

LENOIR M. 1983 - Le Paléolithique des basses vallées de la Dordogne et de la Garonne. Doctorat d'Etat es Sciences, université de Bordeaux I, ex. multigraphié.

LENOIR M. 1987 - La pièce de la Bertonne, « fossile directeur » du Magdalénien ancien ? Bulletin de la Société préhistorique française, t. 84, $\mathrm{n}^{\circ}$ 6, p. 167-171.

LENOIR M., MARMIER F. et TRECOLLE G. 1991 - Données nouvelles sur les industries de SaintGermain-la-Rivière (Gironde), in : 25 ans d'études technologiques en Préhistoire, $\mathrm{XI}^{\mathrm{e}}$ rencontres internationales d'Archéologie et d'Histoire d'Antibes, 1990, Editions APDCA, p. 245-254.

LE TENSORER J.-M. 1981 - Le Paléolithique de l'Agenais. Cahiers du Quaternaire 3.

LEYSALLES G. et NOONE H. V. V. 1949 - Le Pech Saint-Sourd. Bulletin de la Société préhistorique française, t. 53, p. 247-251.

MERLET J.-C. 2005 - Le Badegoulien et le Magdalénien ancien dans le Bassin de l'Adour : un état de la question. Archéologie des Pyrénées occidentales et des Landes, t. 24, p. 103-118.

MILLET-CONTE J.-C. 1994 - Etude de l'industrie lithique du gisement magdalénien de Mirande (commune de Nègrepelisse, Tarn-et-Garonne). Mémoire de Maîtrise, Université de Paris I - Panthéon-Sorbonne, ex. multigraph., $333 \mathrm{p}$.

MILLET-CONTE J.-C. 1995 - Nouvelles données sur le Magdalénien de la vallée de l'Aveyron : une première approche des industries lithiques de la séquence inférieure d'occupation de l'abri Gandil, mémoire de DEA de l'université Paris I - Panthéon-Sorbonne, 172 p.

MORALA A. 1993 - Technologie lithique du Magdalénien ancien de l'abri Casserole ( Les Eyzies de Tayac, Dordogne.). PALEO, 5, Décembre 1993, S.A.M.R.A., Les Eyzies, p. 193-208, 7 fig.

NORMAND Ch. 1986 - Recherches de Préhistoire dans les Landes en 1985, Bulletin de la Société de Borda, $n^{\circ}$ 402, p. 132-140. 
NORMAND Ch. 2002 - Les ressources en matières premières siliceuses dans la basse vallée de l'Adour et de ses affluents. Quelques données sur leur utilisation au Paléolithique supérieur, in : N. Cazals dir., Comportements techniques et économiques des sociétés du Paléolithique supérieur dans le contexte pyrénéen, rapport de P. C. R., 2002, p. 26-38.

ORTEGA I., FOURLOUBEY C., BERTRAN P., GRIGOLETTO F., BEUGNIER V. 2005 - Les Sablons (Marsas, Gironde), rapport final d'opération de fouilles préventives, INRAP Grand Sud-Ouest, SRA d'Aquitaine, $89 \mathrm{p}$.

PARISOT O. 1995 - Analyse typo-technologique du matériel lithique du site d'Orville (Indre, France), Maîtrise de Préhistoire de l'Université de Paris X Nanterre, 2 volumes, 145 p.

PELEGRIN J. 1982 - Approche expérimentale de la technique de production lamellaire d'Orville. In : D. CAHEN éd., Tailler ! Pour quoi faire : Préhistoire et technologie lithique II, Studia Praehistorica Belgica 2, p. 149-158.

PERLES C. 1977 - Note préliminaire sur un type d'outil particulier du site d'Orville (Indre), Bulletin de la Société préhistorique française, t. 72, $\mathrm{n}^{\circ}$ 5, p. 141-144.

PERLES C. 1982 - Les outils d'Orville : des nucléus à lamelles. In : D. CAHEN éd., Tailler ! Pour quoi faire: Préhistoire et technologie lithique II, Studia Praehistorica Belgica 2, p. 129-148.

PERLES C. 1983 - Une technique originale de débitage des lamelles dans le site d'Orville (Indre), Bulletin de la Société préhistorique française, t. 80, c.r.s.m. $n^{\circ}$ 5, p. 98-99.

PESESSE D. 2003 - Approche du comportement technique au Gravettien : l'industrie lithique de l'unité OP10 de la Vigne-Brun, DEA de l'Université de Provence, Aix-Marseille I, 2003.

PESESSE D. 2008 - Les premières sociétés gravettiennes. Analyse comparée des systèmes lithiques de la fin de l'Aurignacien aux débuts du Gravettien. Thèse de doctorat de l'Université d'Aix-en-Provence, ex. multigraphié.

PESESSE D. et MICHEL A. 2006 - Le burin des Vachons : apports d'une relecture technologique à la compréhension de l'Aurignacien récent du nord de l'Aquitaine et des Charentes. PALEO, $\mathrm{n}^{\circ} 18$, Décembre 2006, p. 143-160.

PÉTILLON J.-M, LANGLAIS M., de BEAUNE S.A., BEUKENS R., CHAUVIÈRE F.-X., DAVID F., LETOURNEUX C. et SZMIDT C. 2008 - Le Magdalénien de la grotte des Scilles (Lespugue, HauteGaronne). Premiers résultats de l'étude pluridisciplinaire de la collection Saint-Périer. Antiquités Nationales, $\mathrm{n}^{\circ} 40$, p. 1-15.

PHILIPPE M. et BAZILE F. 2000 - Dynamique interne et aspects technologiques d'une petite série lithique : le niveau $2 \mathrm{~b}$ de l'abri sous roche du Bois-des-Brousses (Herault). Préhistoire et Anthropologie Méditerranéennes, t. 9, p. 5-14.

PRIMAULT J., GABILLEAU J., BROU L., LANGLAIS M. et GUERIN S. 2007 - Le Magdalénien inférieur à microlamelles à dos de la grotte du Taillis des Coteaux à Antigny (Vienne, France), Bulletin de la Société préhistorique française, t. $104, \mathrm{n}^{\circ} 1$, p. 5-30.

RAUX P. et PIEL-DESRUISSEAUX J.L. 1997 - “La cave à endives” ou le Pech del Mas à Cabrerets (Lot), étude

la collection André David. Bulletin de la Société préhistorique française, t. 94, n 1, p. 35-39.

RENARD C. 2002 - Des témoins solutréens en France septentrionale : un mode original de production de support depointe à face plane (La Celle-Saint-Cyr, Yonne), Bulletin de la Société préhistorique française, t. 99, nº 3, p. 461-485. 
RENARD C. 2008 - Les premières expressions du Solutréen dans le Sud-Ouest français. Évolution technoéconomique des équipements lithiques au cours du dernier maximum glaciaire. Thèse de doctorat de l'Université de Paris X -Nanterre, 448 p.

RENARD C. soumis - L'organisation des productions lithiques solutréennes du Cuzoul. Implications techno-économiques In : J. CLOTTES et alii dir., Diversité et identité des groupes solutréens et badegouliens en Quercy : l'exemple de l'abri du Cuzoul de Vers, Lot.

RENARD C. et GENESTE J.-M. 2006 - De la « complexité » des productions lithiques dans le Solutréen supérieur d'Aquitaine. In : L. ASTRUC, F. BON, V. LEA, P.-Y. MILCENT et S. PHILIBERT dir., Normes techniques et pratiques sociales. De la simplicité des outillages pré- et protohistoriques, XXVIe rencontres internationales d'archéologie et d'histoire d'Antibes, 2005, Éd. APDCA, Antibes, p. 119-128.

RIGAUD J.-Ph. dir. 1989 - Le Magdalénien en Europe, Colloque de Mayence, 1987. ERAUL 38.

SACCHI D. 1986 - Le Paléolithique supérieur du Languedoc occidental et du Roussillon, XXIème sup. à Gallia Préhistoire, C.N.R.S, Paris, 284 p., 204 fig., 36 tabl.

SACKETT J. 1999 - The Archaeology of Solvieux. An Upper Palaeolithic open air site in France, Monumenta Archaeologica, 19, Insitute of Archaeology, University of California, Los Angeles, $328 \mathrm{p}$.

SERONIE-VIVEN M., SERONIE-VIVIEN M.-R., FOUCHER P. 2006 - L'économie du silex au Paléolithique supérieur dans le bassin d'Aquitaine. Le cas des silex à lépidorbitoïdes des Pyrénées centrales. Caractérisation et implications méthodologiques, PALEO, 18, Décembre 2006, p. 193-216.

SIMONET A. 2004 -L'atelier de taille gravettien de Tercis (Landes) : approche technologique, économique et sociologique : étude de l'industrie lithique de deux concentrations dites "à pièces à dos" et "à grandes lames”, Mémoire de Maîtrise de l'Université de Toulouse-le Mirail, 204 p.

SORIANO S. 2004 - Pièces d'Orville, de la Bertonne, du Rocher-de-la-Caille... Quelle valeur chronoculturelle pour le Paléolithique supérieur ancien sur la marge sud-est du Bassin parisien ? In : P. BODU, L. CHEHMANA et N. TEYSSANDIER coord., Le Paléolithique supérieur ancien au centre et au sud du Bassin parisien « des systèmes techniques aux comportement, rapport de PCR, Programme P4, Région Centre-Nord, p. 68-77.

SORIANO S et LECHENET H. 2000 - Des “Orvilles” en Bourgogne. L'occupation du site de la Roncière (Bèze, Côte-d'Or) au Paléolithique supérieur. In : G. PION dir., Le Paléolithique supérieur récent : nouvelles données sur le peuplement et l'environnement, table ronde de Chambéry, 1999, Mémoire de la SPF, t. 28, p. 223-233.

TROTIGNON F. 1985 - La station paléolithique de surface de Bellevue. In : Etudes Archéologiques, Crozant, Creuse, Documents d'Archéologie creusoise, nº 1, p. 41-48.

TROTIGNON F. 1993 - Une nouvelle série de Magdalénien aux Venesmes, commune de SaintAmand-Montrond (Cher), Cahiers d'Archéologie et d'Histoire du Berry, n 116, p. 3-14.

TROTIGNON F., POULAIN T., LEROI-GOURHAN Arl. 1984 - Études sur l'abri Fritsch (Indre), XIX ème suppl. à Gallia Préhistoire, CNRS, 122 p.

UTRILLA P. 1981 - El Magdaleniense inferior y medio de la Costa cantabrica, Centro de Investigacion y Museo de Altamira, $n^{\circ} 4$.

UTRILLA P. 1996 - La sistematización del Magdaleniense cantábrico : una revisión histórica de los datos. In : A. MOURRE ROMANILLO éd., "El hombre fósil" 80 anos después, Homenaje a Hugo Obermaier, Ed universidad de Cantabria, p. 211-247. 
VALENTIN B. 1995 - Les groupes humains et leurs traditions au Tardiglaciaire dans le Bassin parisien. Apports de la technologie lithique comparative. Thèse de Doctorat, Université de Paris I PanthéonSorbonne, ex. multigraphié.

\section{ANNEXES}

\section{Abridged english version}

\section{Technological interpretation and discussion around the cultural status of "Bertonne pieces »: the example of Seyresse lithic industry (Landes, France).}

Located at the southern limit of the Sable des Landes (fig. 1), the open-air site of Seyresse was discovered at the end of the 1960's during the clearing of forest undergrowth. The excavation, conducted by R. Arambourou, revealed a Paleolithic camp extending over 80 square meters (Arambourou 1970). Despite a quantitatively limited industry composed of only flint objects (fig. $2, n^{\circ} 2$ ), the spatial distribution of this lithic assemblage is not random and possesses some original characteristics (fig. 3). R. Arambourou judiciously compared some of the tools to "grattoirs de Saint-Sourd" (Saint-Sourd endscrapers) and interpreted then as thinned elements. He thus proposed a Proto-Solutrean attribution of the industry (like that of level $\mathrm{G}$ at Laugerie-Haute Ouest). It was not until the work of M. Lenoir at the site of Bertonne that a new chronological analysis of the site could be realized. This author considers the "pièces de la Bertonne" ("Bertonne pieces"), equivalent to objects found at Seyresse, to be characteristic of an early phase of the Magdalenian (Lenoir 1976 and 1987). Nonetheless, the status of these objects remains unresolved.

In this paper, we favor the often evoked but rarely demonstrated hypothesis that a large number of these pieces could be bladelet cores. Recent studies of industries attributed to the end of the Last Glacial Maximum in France, have shown the importance of the characterization of different bladelet production strategies, which often show cultural distinctions. Through a rapid presentation of the entire assemblage, as well as a detailed analysis of these "Bertonne pieces", we can discuss the place of this type of industry within the chrono-cultural frameworks currently proposed.

Taking advantage of a favorable lithological environment (fig. $2, \mathrm{n}^{\circ} 3$ ), the group(s) that occupied Seyresse preferentially exploited Maastrichtian flints from the Tercis region, located a few km away. The rare pieces probably originating from the Audignon anticline reinforce this evidence for a strictly local exploitation, while no allochthonous elements have been found to indicate extra-regional relations. Though all phases of the core reduction sequence (chaîne opératoire) appear to be represented, a dimensional sorting deprives us of the fine fraction. This bias complicates our understanding of some of the production intentions, particularly those related to bladelet production. Though these factors can limit the precision of some descriptions, a global analysis of the assemblage is still feasible. 
An analysis of the ensemble of retouched pieces shows a diversity of blank forms ranging from fine flakes to elongated flakes to blades. It is nonetheless difficult to relate this diversity to a situation of multiple production strategies (schema opératoires). On the contrary, a preliminary analysis of the cores, along with refits, shows a succession of different objectives applied to the same raw material volume. The laminar productions, perceptible through the existence of carefully made blanks using a soft organic billet (fig. 7, $\mathrm{n}^{\circ} 1$ et 5), remains difficult to determine in its variability. The objective to produce elongated flakes is seen in the industry through the presence of numerous cores (fig. $4, n^{\circ} 3$; fig. $6, n^{\circ} 2$ ) and products with characteristics that are similar to those of blades (dimensions, raw materials) and others that are not (regularity, preparation/percussion). The possibility that these represent, in certain cases, two distinct phases of the same production is illustrated by some cores associating a relatively careful volumetric management (posterior crests, for example) and the utilization of hard stone percussion (fig. $6, \mathrm{n}^{\circ} 1$ ). Most of the fine flakes in the industry seem to have been produced by short series performed at the end of the core reduction sequence in order to produce pieces with sharp edges. For this reason, we do not agree with the attribution by R. Arambourou of some pieces as "raclettes" since the edge retouch of these pieces is not highly characteristic of this tool type. In general, the tool assemblage is composed of burins, most made on blades or elongated flakes (fig. 4, $n^{\circ} 4$ bis; fig. $5, n^{\circ} 2$ ), end scrapers that are sometimes ogival (fig. $9, n^{\circ} 2$ ), perforators and becs (fig. $9, \mathrm{n}^{\circ} 4$ ), rare composite tools (fig. $5, \mathrm{n}^{\circ} 1$ ) and pieces with lateral or denticulate retouch. Some of the unmodified blanks were reintegrated into the production system to be used as bladelet cores.

A precise analysis of the elements considered as "Bertonne pieces" (fig. 7 and 8) enabled us to decompose the different stages of this unique bladelet production strategy (fig. 10). The selection of elongated blanks is logical in the choice of the orientation of the flaking surface-distal, proximal or both (fig. 10-1). Ideally, the voluntary segmentation of these regular volumes results in the creation of several volumes with nearly identical characteristics. This segmentation plays an important role during the phases of opening or restoring a bladelet surface through the creation of an exploitable ridge (fig. 10-2A). This surface can also be regularized by a partial or total, inverse "truncation" ( $2 \mathrm{~B}$ and $\mathrm{C}$ ). The striking platform, sometimes constituted by a facet of the superior face can also be created by direct "retouch" of the lateral edge. In most of the unique cases, it is lateralized on the left. The production sequence consists of the detachment of a first long bladelet along the ridge (3A and $\mathrm{B})$. The negative thus created can be used for the detachment of a second bladelet (3C). Though they exist, re-modifications of the opposite end are rare (3D). Debitage under these conditions cannot continue without a restoration of the flaking surface, either by fracturation, or by the direct realization of a new inverse truncation, both of which produce characteristic waste products.

This particular production strategy is used almost exclusively to obtain fine bladelets that are usually straight in profile, or sometimes slightly twisted toward the proximal end. According to the last visible bladelet removal scars, these pieces seldom exceed 30 $\mathrm{mm}$ in length. The methods(s) used to produce these blanks remains to be determined. In summary, it involves a debitage operation along the edge of the blank, which is transversally oriented, with characteristic removals that intentionally overlap onto the inferior/ventral face. 
While the most recent publications concerning the Gravettian and Solutrean of southern France allow us, a priori, to eliminate the hypothesis of an attribution to these cultures, a Magdalenian attribution can still be considered. In the earliest phase of this cultural entity, we observe assemblages dominated by microbladelet productions, one of which has been morphologically compared to "Bertonne pieces" (fig. 12, $\mathrm{n}^{\circ}$ 1-3). Meanwhile, this production type, described as being "on an off-centered (déjeté) ventral front" shows rather clear differences from the Seyresse pieces, including the absence of modification by inverse truncation, the type of striking platform and often recurrent debitage rhythm. Moreover the "off-center ventral front" debitage of the Lower Magdalenian is one of a group of strategies with the objective of producing microbladelets backed on the right side and with a convex cutting edge (Langlais 2007).

Finally, it is indeed in the Badegoulian that we can observe much clearer links. A comparison of the lamellar scheme of Seyresse with the "transverse burins" of the Badegoulian (Bodu et al. 2007) suggests a conceptual relationship between these two strategies (fig. 12, $\mathrm{n}^{\circ} 8$ and 9 ). This technological link is also supported by the existence within the Seyresse industry of two pieces that belong to the "preferential core edge" debitage family (Ducasse et Langlais 2007) made on "transverse burins on notched pieces" (fig. 13).

The attribution of this assemblage to the Badegoulian raises new questions concerning the status of such assemblages at the scale of this entity. Indeed, the framework established for this techno-complex favors a chronological division based in large part on the variability of certain lithic elements (raclettes and transverse burins). Though we do not question the Early to Late Badegoulian succession, we must note that an assemblage such as that of Seyresse does not fit into this framework. Which Badegoulian is it? With few arguments pleading in favor of a chronological interpretation, other hypotheses must be considered (site type, site function, etc.).

\section{NOTES}

4. «Malgré ses caractères originaux et l'absence d'autres éléments de datation sur ce sondage, il semble que cette industrie puisse être attribuée à un Magdalénien. » (Perlès 1983).

5. Notons que les observations effectuées par l'un de nous (M. L.) sur la série lithique de La Marche y ont montré l'absence de " pièces d'Orville ».

6. Notons qu'un support rebroussé présente une probable tentative d'extraction lamellaire aux dépens

de cette convexité « naturelle ».

7. Bordes J.-G., communication inédite - Burins à Caminade, au Roc-de-Combe et au Piage : répartition diachronique, variabilité des formes... et des fonctions? Table Ronde internationale d'Aix-en-Provence, 3-5 mars 2003.

8. Cet aspect devra faire l'objet d'analyses ultérieures sur des corpus plus étendus.

9. Notons par ailleurs que cette association existe également sur certains nucléus de type «La Marche » du site éponyme (obs. pers.).

10. Évoquant une simple morphologie, l'appellation «lamelle à pan cannelé », bien que discutable, nous semble d'usage plus adéquat que les expressions "lamelle à pan facetté » et " lamelle d'Orville », la première parce qu'elle introduit un terme descriptif déjà consacré à un autre usage, la seconde pour ce qu'elle véhicule implicitement (terme éponyme). 
11. Celui-ci réunit à lui seul plus de $45 \%$ du nombre total de pièces de La Bertonne publiées à ce jour.

12. Variabilité qui, au-delà de cette question, reste une réalité des assemblages badegouliens (e.g. Fourloubey 1996 ; Cretin 2000).

13. Il existe bien entendu des termes de passage entre nucléus "sur front dorsal », «sur front ventral déjeté » et sur « tranche transversale » (fig. 12, $\mathrm{n}^{\circ} 2$ ), ceci renforçant leur statut de simple modalité (Langlais 2007).

14. Les contextes de récolte de la grande majorité des sites à «Bertonnes » n'ayant pas, à ce jour, permis la mise en évidence des objectifs liés à ces nucléus.

15. Les recherches en cours sur cet ensemble (en collaboration avec J.-C. Castel et F.-X. Chauvière) sembleraient indiquer qu'il puisse s'agir d'un matériel provenant d'anciennes « opérations » menées sur le site voisin du Petit Cloup Barrat (Castel et al. 2006).

16. Notons que P.-Y. Demars décompte un nombre relativement important de raclettes à la Bombetterie $(\mathrm{N}=44)$. Cependant, outre des dessins peu convaincants quant à la nature de ces pièces (éclats retouchés?), il paraît étonnant que A. Cheynier, l'un des «découvreur " de la raclette (Cheynier 1930), n'en fasse aucune mention lors de la publication du matériel qu'il a lui même récolté (Cheynier 1956).

17. D'autant qu'il existe au moins une lamelle à «pan cannelé » au sein de l'industrie (Ducasse, thèse en cours).

18. L'un de nous (S. D.), accompagné de P. Bodu et L. Chehmana, a en effet pu observer ces pièces sur l'invitation de C. Cretin (que nous souhaitons remercier ici) dans le cadre d'une discussion commune autour des industries de Badegoule (novembre 2005 au M. A. N.).

19. Dans cette perspective, une tentative de localisation des déblais de la fouille de Seyresse ne semble pas illusoire (c. Normand com. pers.), ce qui pourrait permettre d'accéder «physiquement » aux éléments jusqu'ici inférés et ce, de façon à contrôler la validité d'une partie des hypothèses développées.

\section{RÉSUMÉS}

Le site de plein air de Seyresse (Landes, France), fouillé à la fin des années soixante par R. Arambourou, a livré une série lithique originale bien que quantitativement faible. Rapproché lors de sa publication au Proto-Solutréen, cet assemblage a fait l'objet, dès le milieu des années quatre-vingt, de nouvelles expertises conduisant à une révision de cette interprétation chronoculturelle. En effet, certaines pièces qui, comportant des enlèvements plans inverses d'extrémité avaient été rapprochées d'outils solutréens, correspondent plus vraisemblablement aux « pièces de la Bertonne » décrites par M. Lenoir à partir de sites girondins attribués pour leur plus grande part au Badegoulien/Magdalénien ancien (0/1). Les données acquises ces dernières années par le biais des travaux menés sur les industries lithiques comprises entre 19500 et 15000 BP dans la moitié sud de la France sont venues préciser le cadre chronologique post-solutréen (validant notamment une succession Badegoulien à raclettes/Magdalénien inférieur), permettant alors d'appréhender ces industries à " pièces de la Bertonne " sous un angle nouveau. À la suite d'une présentation générale de l'industrie de Seyresse, une lecture technologique de ces éléments particuliers est proposée, conduisant à confirmer une hypothèse souvent évoquée, consistant à les intégrer au sein d'un schéma opératoire de production de lamelles. Si les 
comparaisons effectuées nous permettent, en l'état actuel des données, de rejeter l'hypothèse magdalénienne, elles conduisent à privilégier l'attribution de la série au Badegoulien.

\section{INDEX}

Keywords : Badegoulian, bladelet production, End of the Last Glacial Maximum, lithic industries, Lower Magdalenian, pièces de la Bertonne, pièces d'Orville

Mots-clés : Badegoulien, fin du Dernier Maximum Glaciaire, industries lithiques, Magdalénien inférieur, pièces de la Bertonne, pièces d'Orville, production lamellaire

\section{AUTEURS}

\section{SYLVAIN DUCASSE}

Laboratoire TRACES (UMR 5608, UTAH), Maison de la recherche, 5 allées Antonio Machado, 31058 Toulouse Cedex 9 - sylvain.ducasse@orange.fr

\section{MATHIEU LANGLAIS}

Laboratoire TRACES (UMR 5608, UTAH), Maison de la recherche, 5 allées Antonio Machado, 31058

Toulouse Cedex 9 et SERP, université de Barcelone, matlang1@wanadoo.fr 